\title{
Ultrashort Laser Pulses and Applications
}

Edited by W. Kaiser

With Contributions by

D.H. Auston K.B. Eisenthal R.M. Hochstrasser

C.K. Johnson W. Kaiser A. Laubereau

D. von der Linde A. Seilmeier C.V.Shank W.Zinth

With 196 Figures

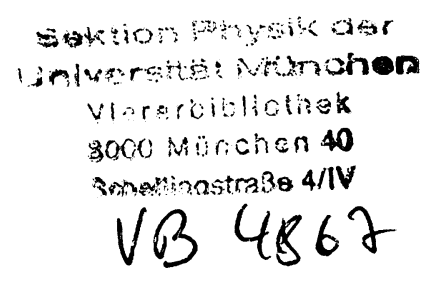

Springer-Verlag Berlin Heidelberg New York London Paris Tokyo 


\title{
Professor Dr. Wolfgang Kaiser
}

Physik Department E11, Technische Universität, Arcisstr. 21

D-8000 München 2, Fed. Rep. of Germany

\section{Managing Editor:}

\author{
Dr. Helmut K. V. Lotsch
}

Springer-Verlag, Tiergartenstraße 17

D-6900 Heidelberg, Fed. Rep. of Germany

\section{ISBN 3-540-18605-0 Springer-Verlag Berlin Heidelberg New York ISBN 0-387-18605-0 Springer-Verlag New York Berlin Heidelberg}

Library of Congress Cataloging-in-Publication Data. Ultrashort laser pulses and applications. (Topics in applied physics; v. 60) Includes bibliographies and index. 1. Laser pulses, Ultrashort. I. Kaiser, W. (Wolfgang), 1925 - . II. Auston, D. H. III. Series. QC689.5.L37U469 $1988 \quad 621.36$ '6 $88-2009$

This work is subject to copyright. All rights are reserved, whether the whole or part of the material is concerned, specifically the rights of translation, reprinting, reuse of illustrations, recitation, broadcasting, reproduction on microfilms or in other ways, and storage in data banks. Duplication of this publication or parts thereof is only permitted under the provisions of the German Copyright Law of September 9, 1965, in its version of June 24,1985 , and a copyright fee must always be paid. Violations fall under the prosecution act of the German Copyright Law.

(C) Springer-Verlag Berlin Heidelberg 1988

Printed in Germany

The use of registered names, trademarks, etc. in this publication does not imply, even in the absence of a specific statement, that such names are exempt from the relevant protective laws and regulations and therefore free for general use.

Typesetting: Asco Trade Typesetting Limited, Hong Kong

Printing and binding: Brühlsche Universitätsdruckerei, Giessen

$2154 / 3150-543210$ 


\section{Contents}

1. Introduction. By W. Kaiser . . . . . . . . . . . . . . . . 1

1.1 Organisation of the Book . . . . . . . . . . . . . . . 2

2. Generation of Ultrashort Optical Pulses

By C. V. Shank (With 20 Figures) . . . . . . . . . . . . . 5

2.1 Introduction . . . . . . . . . . . . . . . . . . . 5 5

2.2 Mode-locking . . . . . . . . . . . . . . . . . . 5

2.2.1 Basic Concepts . . . . . . . . . . . . . . . . . 5

2.2.2 Active Mode-locking . . . . . . . . . . . . . . 7

2.2.3 Synchronous Pumping . . . . . . . . . . . . . . 8

2.2.4 Passive Mode-locking . . . . . . . . . . . . . . 9

a) Type 1: Giant-Pulse Lasers . . . . . . . . . . 10

b) Type 2: Continuous Lasers . . . . . . . . . . 11

2.3 Short Pulse Laser Systems . . . . . . . . . . . . . . . 13

2.3.1 Dye Lasers . . . . . . . . . . . . . . . . . . . 13

a) Synchronous Mode-locking . . . . . . . . . . 13

b) Passive Mode-locking . . . . . . . . . . . . . . 15

c) Hybrid Mode-locking Techniques . . . . . . . . 18

d) Amplification . . . . . . . . . . . . . . . . . . . . 19

2.3.2 Color Center Lasers . . . . . . . . . . . . . . . 22

2.3.3 Novel Pulse Generation Techniques . . . . . . . . 23

2.3.4 Diode Lasers . . . . . . . . . . . . . . . . . . 24

2.4 Pulse Compression . . . . . . . . . . . . . . . . . . 25

2.4.1 Theory . . . . . . . . . . . . . . . . . 26

2.4.2 Compression Experiments . . . . . . . . . . . . 29

References . . . . . . . . . . . . . . 31

3. Optical Nonlinearities with Ultrashort Pulses

By A. Laubereau (With 27 Figures). . . . . . . . . . . . . 35

3.1 Nonlinear Polarization . . . . . . . . . . . . . . . . 35

3.2 Three-Wave Interactions . . . . . . . . . . . . . . . 36

3.2.1 Second Harmonic Generation . . . . . . . . . . . 36

3.2.2 Sum-Frequency Generation and Its Application for UV Pulses . . . . . . . . . . . . . . . . . . 42

3.2.3 Difference-Frequency Generation and Down-Conversion to the Far Infrared . . . . . . 45 
3.2.4 Stimulated Parametric Interaction . . . . . . . . . 47

a) Stimulated Parametric Generators . . . . . . . 49

b) Optical Parametric Amplifiers . . . . . . . . . . . 50

c) Synchronously Coupled Parametric Oscillators . . 51

d) Stimulated Parametric Emission in Extended Spectral Regions . . . . . . . . . . . . . . . 52

3.2.5 Further Applications . . . . . . . . . . . . . . 53

a) Second Harmonic Probing . . . . . . . . . . . . 53

b) Ultrafast Parametric Light Gates . . . . . . . . 54

c) Analysis of Pulse Shape . . . . . . . . . . . . . 56

3.3 Four-Wave Interactions . . . . . . . . . . . . . . . . 60

3.3.1 Sum-Frequency Generation . . . . . . . . . . . . 60

a) Third and Higher Order Harmonic Generation . . 60

b) Four-Wave Up-Conversion . . . . . . . . . . 62

3.3.2 Four-Wave Difference-Frequency Mixing . . . . . . 63

a) General Discussion . . . . . . . . . . . . . . 64

b) Degenerate Four-Wave Mixing (DFWM) . . . . 66

3.3.3 Stimulated Four-Wave Interactions . . . . . . . . 68

a) Stimulated Four-Wave Parametric Emission . . . 69

b) Optical Phase Conjugation . . . . . . . . . . . . 70

c) Stimulated Raman Scattering and Related Raman Interactions . . . . . . . . . . . . . . 73

3.3.4 Further Four-Wave Interactions . . . . . . . . . . 83

a) Induced Population Gratings . . . . . . . . . . . 83

b) The Coherence Peak . . . . . . . . . . . . . . . . . . . 88

c) Continuum Generation . . . . . . . . . . . . . 90

d) Ultrafast Bistability and Hysteresis . . . . . . . 91

3.4 Short Pulse Nonlinearities with Special Boundary Conditions 94

3.4.1 Nonlinear Effects at Interfaces . . . . . . . . . . 94

3.4.2 Quantum Size Effects in Nonlinear Optics . . . . . 97

3.4.3 Nonlinear Pulse Propagation in Optical Fibers . . . 98

3.5 Conclusions . . . . . . . . . . . . . . . . . . . . . 101

References .................. . 102

\section{Ultrashort Interactions in Solids}

By D. von der Linde (With 42 Figures) . . . . . . . . . . . 113

4.1 Exciton Dynamics . . . . . . . . . . . . . . . . . . 114

4.1.1 Exciton-Polariton Dispersion . . . . . . . . . . . 115

4.1.2 Exciton Relaxation . . . . . . . . . . . . . . . 119

4.1.3 Exciton Screening . . . . . . . . . . . . . . . . 123

4.2 Electron-Hole Dynamics . . . . . . . . . . . . . . . 129

4.2.1 Electron-Hole Plasma . . . . . . . . . . . . . . 130

4.2.2 Carrier Thermalization . . . . . . . . . . . . . . 137

4.2.3 Electronic Relaxation in Disordered Materials and Quantum Wells . . . . . . . . . . . . . 142 
4.3 Phonon Dynamics . . . . . . . . . . . . . . . . . . 146

4.3.1 Optical Phonon Lifetimes . . . . . . . . . . . . 149

4.3.2 Relaxation of Incoherent Phonons . . . . . . . . . 152

4.3.3 Multiphonon States . . . . . . . . . . . . . . . 156

4.4 Ultrafast Phase Transformations . . . . . . . . . . . . 159

4.4.1 Amorphization . . . . . . . . . . . . . . . . . 161

4.4.2 Surface Melting . . . . . . . . . . . . . . . . . 164

4.4.3 Density of Electrons and Holes . . . . . . . . . . 169

4.4.4 Electron and Lattice Temperature . . . . . . . . . 172

4.5 Concluding Remarks . . . . . . . . . . . . . . . . . 177

References . . . . . . . . . . . . . . . . . . 178

5. Ultrafast Optoelectronics. By D. H. Auston (With 26 Figures) . 183

5.1 Introduction . . . . . . . . . . . . . . . . . . . . . 183

5.2 Optoelectronic Device Concepts . . . . . . . . . . . . 185

5.3 Materials for Ultrafast Optoelectronics . . . . . . . . . 188

5.3.1 Photoconducting Materials . . . . . . . . . . . . 188

a) Intrinsic Speed of Response of Semiconductors . . 189

b) High Electric Field Effects . . . . . . . . . . . . 191

c) Trapping and Recombination . . . . . . . . . . 191

d) Radiation Damage . . . . . . . . . . . . . . . . 192

e) Carrier Mobilities . . . . . . . . . . . . . . . . 193

f) Other Photoconducting Materials . . . . . . . . 194

5.3.2 Electro-optic Materials . . . . . . . . . . . . . . 195

a) Selection of Electro-optic Materials for

Picosecond Optoelectronics . . . . . . . . . . 196

5.4 Generation and Detection of Ultrashort Electrical Pulses . 198

5.4.1 Picosecond Photoconductors . . . . . . . . . . . 198

a) Response of an Ideal Photoconductor in a
Transmission Line . . . . . . . . . . . . 200

b) Photoconducting Electrical Pulse Generators . . . 200

c) Photoconducting Electronic Sampling . . . . . . 202

d) Electronic Autocorrelation Measurements . . . . 203

5.4.2 Electro-optic Devices . . . . . . . . . . . . . . 204

a) Electro-optic Sampling . . . . . . . . . . . . 204

b) Optical Rectification . . . . . . . . . . . . . . . 207

c) Electro-optic Cherenkov Radiation . . . . . . . 208

5.4.3 Ultrafast Electrical Transmission Lines . . . . . . . 209

5.5 Optoelectronic Measurement Systems and Their Applications 211

5.5.1 Optoelectronic Measurement Systems . . . . . . . 211

a) Sensitivity . . . . . . . . . . . . . . . . . . . . 212

b) Timing Accuracy . . . . . . . . . . . . . . . . . 212

c) Non-invasive Probing . . . . . . . . . . . . . . 212

d) Low Temperature Environments . . . . . . . . 212 
e) High Power . . . . . . . . . . . . . . . . . . . . 213

f) Simultaneous Time and Frequency Measurements. 213

g) Electrical Isolation . . . . . . . . . . . . 213

5.5.2 Characterization of High Speed Discrete Devices . . 214

a) Photoconductive Sampling of High Speed Photodetectors .

b) Electro-optic Sampling of High Speed Photodetectors . . . . . . . . . . . . . . . 214

c) Optical Mixing in Photodetectors . . . . . . . . . 214

d) Impulse Response of High Speed Transistors . . . 215

e) Picosecond Photoemissive Sampling . . . . . . . 217

5.5.3 Optoelectronic Measurements of Integrated Circuits . 217

a) Substrate Probing of GaAs Integrated Circuits . . 218

b) A Picosecond Electro-optic Wafer Prober . . . . 218

c) Charge Density Probing in Integrated Circuits . . 219

d) Direct Optical Stimulation of Integrated Circuits . 219

e) Integrated Photoconductors . . . . . . . . . . 220

5.5.4 Microwave, Millimeter-Wave, and Far-Infrared Applications . . . . . . . . . . . . . . . . . . 221

a) Photoconductive Switching and Gating . . . . . 221

b) Optical Rectification . . . . . . . . . . . . . 222

c) Phase Modulation of Microwave and Millimeter Waves . . . . . . . . . . . . . . 223

d) Radio Frequency Mixing . . . . . . . . . . . 223

e) Photoconducting Antennas . . . . . . . . . . . 224

5.5.5 High Power Optoelectronic Switches . . . . . . . . 226

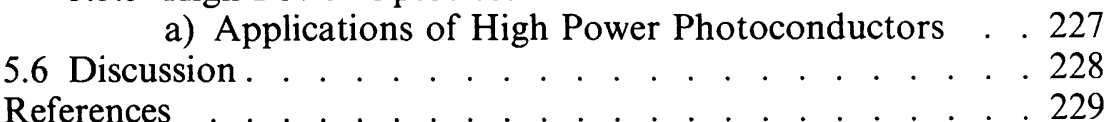

\section{Ultrafast Coherent Spectroscopy}

By W. Zinth and W. Kaiser (With 20 Figures) . . . . . . . . 235

6.1 Theory of Time-Resolved Coherent Spectroscopy . . . . . 236

6.1.1 The Theoretical Model . . . . . . . . . . . . . . 236

6.1.2 Equations of Motion . . . . . . . . . . . . . . 237

6.1.3 Ultrafast Coherent Techniques . . . . . . . . . . 239

a) One Excitation Pulse . . . . . . . . . . . . . . 239

b) Two Excitation Pulses. . . . . . . . . . . . . . 240

c) Three Excitation Pulses . . . . . . . . . . . . . . . . . . . 241

6.1.4 Electric Dipole and Raman-Type Transitions . . . . 241

a) Electric Dipole Transitions. . . . . . . . . . . 241

b) Raman-Type Transitions . . . . . . . . . . . . . 242

c) Pulse Area and Population Changes in Ultrafast Coherent Experiments . . . . . . . . . . . 243 
6.2 Coherent Spectroscopy Using a Single Excitation Process

6.2.1 Time-Resolved Coherent Raman Spectroscopy of a Single Homogeneously Broadened Transition . . . . 244

a) Experimental Arrangements . . . . . . . . . . 246

b) Dephasing in Liquids . . . . . . . . . . . . . . 247

c) Vibrational Transitions in Solids . . . . . . . . 251

d) The Collision Time . . . . . . . . . . . . . . . 255

6.2.2 Time-Resolved Coherent Raman Scattering of a

Distribution of Resonance Frequencies . . . . . . . 256

a) Time-Domain Experiments . . . . . . . . . . 256

b) Transient Frequency-Domain Experiments . . . . 259

6.2.3 Resonant Pulse Propagation in the Infrared . . . . . . 262

6.3 Coherent Spectroscopy Using Multiple Excitation Processes 264

6.3.1 Ultrafast Two-Pulse Echo Spectroscopy . . . . . . 265

6.3.2 Three-Pulse Echos . . . . . . . . . . . . . . . . 267

a) Measurements of Energy Relaxation Times . . . 269

b) Measurements of Dephasing Times . . . . . . . 270

c) Accumulated Three-Pulse Echos . . . . . . . . 271

d) Two-Color Echos. . . . . . . . . . . . . . . . 271

6.4 Summary . . . . . . . . . . . . . . . . . 273

6.5 Appendix . . . . . . . . . . . . . . . . . . . . . 273

References . . . . . . . . . . . . . . . 274

7. Ultrashort Intramolecular and Intermolecular Vibrational

Energy Transfer of Polyatomic Molecules in Liquids

By A. Seilmeier and W. Kaiser (With 22 Figures) . . . . . . . 279

7.1 Experimental Techniques . . . . . . . . . . . . . . . 280

7.1.1 Excitation . . . . . . . . . . . . . . . . . 280

7.1 .2 Probing . . . . . . . . . . . . . . . . . . . 282

7.1.3 Experimental System . . . . . . . . . . . . . . 284

7.2 Vibrational Population Lifetimes $T_{1}$ of Small Molecules

in the Electronic Ground State $S_{0}$. . . . . . . . . . . 285

7.2.1 Experimental Data . . . . . . . . . . . . . . . 286

7.2.2 Theoretical Interpretation and Examples . . . . . . 290

a) $\mathrm{CH}_{2}=\mathrm{CCl}_{2}$. . . . . . . . . . . . . . 292

b) trans $-\mathrm{CHCl}=\mathrm{CHCl}$. . . . . . . . . . . . . 293

7.3 Vibrational Redistribution in Large Molecules

in the Ground State $S_{0}$. . . . . . . . . . . . . . . . . 294

7.3.1 The Redistribution Time . . . . . . . . . . . . . 295

7.3.2 The Vibrational Distribution . . . . . . . . . . . 297

7.3.3 The Concept of Internal Temperature . . . . . . . 301

7.4 Intermolecular Transfer of Vibrational Energy of Large

Molecules to the Surrounding Solvents . . . . . . . . . 303

7.4.1 Molecular Excitation with Infrared Pulses . . . . . 304 
7.4.2 Molecular Excitation via Rapid Internal Conversion . 305

7.4.3 Discussion and Model for Energy Transfer . . . . . 306

7.5 Equalization of Energy in Liquids Measured with a

Molecular Thermometer . . . . . . . . . . . . . . . . 309

7.5.1 The Molecular Thermometer . . . . . . . . . . . 309

7.5.2 Energy Transfer Processes . . . . . . . . . . . . 310

7.5.3 Experimental Observations . . . . . . . . . . . . 311

7.6 Intramolecular Vibrational Relaxation of Large Molecules

in the First Excited Electronic State $S_{1}$. . . . . . . . . 314

References . . . . . . . . . . . . . . 315

\section{Ultrafast Chemical Reactions in the Liquid State}

By K. B. Eisenthal (With 16 Figures) . . . . . . . . . . . . . 319

8.1 Introduction . . . . . . . . . . . . . . . . . . . . . 319

8.2 Fast Chemical Reactions - Solvent Effects . . . . . . . . 319

8.3 Photoisomerization . . . . . . . . . . . . . . . . . . 320

8.3.1 Kramers Model of a Chemical Reaction . . . . . . 321

8.3.2 Photoisomerization at Low Densities. . . . . . . . 323

8.3.3 Photoisomerization of Polar Molecules . . . . . . . . 325

8.3.4 Photoisomerization with Femtosecond Pulses . . . . 327

8.4 Photodissociation and Recombination of Molecular Iodine in Solution . . . . . . . . . . . . . . . . . . . . . 328

8.5 Chemical Intermediates . . . . . . . . . . . . . . . . . . 329

8.5.1 Role of Charge Transfer in Chemical Reactions . . . 329

a) Hydrogen Atom Transfer by Electron Plus Proton Transfer. . . . . . . . . . . . . . 330

b) Electron Donor-Acceptor Complexes . . . . . . 332

8.5.2 Carbenes . . . . . . . . . . . . . . . . 333

a) Spin Relaxation in Carbenes - Solvent and Structure Effects . . . . . . . . . . . . . . . . 334

b) Photochemistry of Chemical Intermediates . . . 336

8.5.3 Nitrenes . . . . . . . . . . . . . . . . . . . . 337

8.5.4 Photofragmentation of Singlet Oxygen Precursors . . 338

8.5.5 Biradicals . . . . . . . . . . . . . . . . . . . 339

8.6 Excited-State Proton Transfer . . . . . . . . . . . . . 341

8.6.1 The Naphthols - Intermolecular Proton Transfer . . 342

8.6.2 Intramolecular Proton Transfer . . . . . . . . . . 343

a) Transfer in Non-Hydrogen-Bonding Solvents . . . 343

b) Transfer in Hydrogen-Bonding Solvents . . . . . 345

c) Double Proton Transfer . . . . . . . . . . . . . . 348

8.7 Reactions in Bulbs and Beams . . . . . . . . . . . . . 349

8.8 Concluding Remarks . . . . . . . . . . . . . . . . . 350

References . . . . . . . . . . . . . . . 350 
9. Biological Processes Studied by Ultrafast Laser Techniques

By R. M. Hochstrasser and C. K. Johnson (With 23 Figures) . . 357

9.1 Heme Proteins . . . . . . . . . . . . . . . . . . . 357

9.1.1 Optical Spectroscopy of Heme Proteins . . . . . . 359

9.1.2 Structural Information . . . . . . . . . . . . . 363

9.1.3 Transient Raman Spectroscopy. . . . . . . . . . 364

9.1.4 Picosecond Transient Infrared Spectroscopy . . . . 367

9.1.5 Protein Dynamics . . . . . . . . . . . . . . . 367

9.1.6 Fluorescence of Tryptophans in $\mathrm{Mb}$ and $\mathrm{Hb}$. . . . 368

9.1.7 Molecular Dynamics Simulations . . . . . . . . . 372

9.1.8 Geminate Recombination . . . . . . . . . . . . 374

9.1.9 Photophysics of Hemes and Heme Proteins . . . . 375

9.1.10 Laser Induced Heating . . . . . . . . . . . . . 377

9.1.11 Summary . . . . . . . . . . . . . . . . . . . 380

9.2 Photosynthesis . . . . . . . . . . . . . . . . . . . . 380

9.2.1 Electron Transfer . . . . . . . . . . . . . . . . 381

9.2.2 Bacterial Reaction Centers . . . . . . . . . . . 384

9.2.3 Antenna Energy Transfer . . . . . . . . . . . . 390

9.2.4 Reaction Centers in Green Plants . . . . . . . . . 395

9.3 Rhodopsin and Bacteriorhodopsin . . . . . . . . . . 396

9.3.1 Introduction and Background . . . . . . . . . . 396

9.3.2 Rhodopsin and Bacteriorhodopsin Photochemistry . 396

9.3.3 Picosecond Experiments . . . . . . . . . . . . . 401

a) Visual Pigments . . . . . . . . . . . . . . . . . . . . 401

b) Purple Membrane Pigments . . . . . . . . . . . 404

9.3.4 Summary . . . . . . . . . . . . . . . . . . . 409

References . . . . . . . . . . . . . . . 409

Subject Index . . . . . . . . . . . . . . . . . . . . . . . 419 



\title{
6. Ultrafast Coherent Spectroscopy
}

\author{
Wolfgang Zinth and Wolfgang Kaiser
}

With 20 Figures

Coherent spectroscopy, initially started in the mid-sixties, is intimately conncected with the development of intense coherent light sources. The generation of ultrashort light pulses enabled coherent spectroscopy to be extended to real-time measurements of rapid dynamic processes on the time scale of $10^{-12} \mathrm{~s}$. The early measurements of picosecond time-resolved coherent Raman scattering in 1971 were followed by numerous time-resolved techniques adapted to a variety of ultrafast phenomena. Most investigations are concerned with rapid dynamic processes in the condensed phases. The experimental progress stimulated theoretical investigations, improving our understanding of the interactions in liquids and solids. In recent years, a number of dynamic relaxation processes have been elucidated.

The title of this chapter, Ultrafast Coherent Spectroscopy, requires some explanation since it implies investigations in the time and frequency domain. Actually, it adequately characterizes the present situation. Coherent techniques give information on dynamic processes in the time domain as well as on linewidths and line positions in the frequency domain.

Numerous ultrafast investigations are concerned with coherent Raman scattering by molecular levels in the electronic ground state. The experimental techniques are well established on the picosecond time scale. Currently, these experiments are being extended to the study of even faster processes in the femtosecond time domain. A substantial fraction of coherent experiments deal with the study of vibrational levels in excited electronic states. Here, echo and induced-grating experiments have been successfully applied to reveal the relaxation dynamics.

The present chapter is organized as follows: In Sect. 6.6.1 the theoretical background of ultrafast coherent spectroscopy is given together with a short description of dynamical processes in condensed matter. Section 6.2 reports on time-resolved investigations using a single excitation process. Various time resolved coherent Raman and infrared techniques are discussed. Finally, in Sect. 6.3 echo-type experiments, i.e. experimental techniques with multiple excitation are presented. 


\subsection{Theory of Time-Resolved Coherent Spectroscopy}

This section deals with the principles of time-resolved coherent experiments in a semi-classical approach. The electromagnetic light field is treated classically, the molecular system quantum-mechanically by its density matrix. Dynamic interaction processes between molecules have two important relaxation times - the phase relaxation time $T_{2}$ and the energy relaxation time $T_{1}$. We shall repeatedly refer to the relationship between time-domain coherent experiments and frequency-domain steady-state measurements.

\subsubsection{The Theoretical Model}

Coherent spectroscopy is presented here as a tool to investigate transitions between different energy levels or - more precisely - to study the transition frequencies and the relaxation processes related to these energy states. The essential features of the theoretical model are:

i) Of the many molecular transitions we treat only two levels, the ground state $|b\rangle$ and the excited state $|a\rangle$ separated by the energy $E=\hbar \omega_{0}$. All other states are assumed not to interact with the electromagnetic excitation and probing fields. This approach is well justified for most molecular systems [6.1].

ii) We assume that each molecular two-level system is only weakly interacting with the other molecules which are assumed to act as a fluctuating bath [6.2-6]. The Hamiltonian $H$ of the molecule is written as a sum of an unperturbed Hamiltonian $H_{0}$ and two interaction Hamiltonians, the latter are: $H_{\mathrm{im}}$ which gives the interaction between the molecules and $H_{\mathrm{ie}}$ characterizing the interaction of the molecules with the electromagnetic fields [6.7,8]. The molecular interaction consists of a static contribution which leads to a constant frequency shift, and of a fluctuating contribution which modulates the resonance frequency and leads to a broadening of the transition band. The microscopic nature of the dynamic interaction may be understood in liquids in terms of "collisions" between individual molecules [6.2,4]. The collision process will be treated in detail in the context of Sect. 6.2.1. A major interaction in solids is caused by the thermal distribution of the acoustic phonons. In the macroscopic ensemble of two-level systems the dynamic interaction leads to relaxation time constants in the equation of motion (see below).

iii) The ensemble of two-level systems is described by the density matrix $\rho$ with the diagonal elements $\rho_{a a}$ and $\rho_{b b}$ and the off-diagonal elements $\rho_{a b}=\rho_{b a}^{*}$. The density matrix follows the equation of motion

$$
\frac{\partial \rho}{\partial t}=\frac{\mathrm{i}}{\hbar}[\rho, H] \text {. }
$$

iv) The electromagnetic field is treated classically, since the number of photons is quite large in most coherent experiments. We work with plane waves, i.e. 
the fields have the form: $E=E_{0} \cos (\Omega t+k r)$ and consider electric dipole interactions with the Hamiltonian $H_{\mathrm{ie}}=\hat{\mu} E(t) . \hat{\mu}$ is the component of the dipole operator along the direction of the electric field $E$ with $\mu_{a a}=\mu_{b b}=0$ and $\mu_{b a}=\mu_{a b}=\mu$.

The evolution of the electromagnetic fields is described by the wave equation

$$
\Delta E-\frac{n^{2}}{c^{2}} \frac{d^{2} E}{d t^{2}}=\frac{1}{\varepsilon_{0} c^{2}} \frac{d^{2} P^{\mathrm{NL}}}{d t^{2}} .
$$

The source term on the right-hand side of (6.2) describes the generation of light fields. The nonlinear polarization is proportional to the macroscopic dipole moment, $P=N\langle\hat{\mu}\rangle=N \times \operatorname{Tr}\{\hat{\mu} \rho\}=N \mu\left(\rho_{a b}+\rho_{b a}\right)$, where $N$ is the number of molecules per unit volume. Thus the radiation intensity observed in the coherent experiment is proportional to $|N \operatorname{Tr}\{\hat{\mu} \rho(t)\}|^{2}$.

\subsubsection{Equations of Motion}

Here we discuss the equations for the different components of the density matrix. The system of equations can be solved in a formal way by simple multiplication of matrices. In this way it is possible to readily see the salient features of the coherent spectroscopy. The discussion presented here follows a treatment given by Hesselink and Wiersma [6.9].

The equation of motion (6.1) is rewritten in (6.3-5) for the different density matrix elements. The electric field is coupled to the molecules via the interaction Hamiltonian $H_{\mathrm{ie}}=-\hat{\mu} E(t)$. It is convenient to introduce $\tilde{\rho}_{b a}=\rho_{b a} \exp (\mathrm{i} \Omega t)$ and to apply the rotating frame approximation.

$$
\begin{aligned}
& \dot{\rho}_{a a}=\frac{\mathrm{i} \mu E_{0}}{2 \hbar}\left(\tilde{\rho}_{b a} \mathrm{e}^{-\mathrm{i} k r}-\tilde{\rho}_{a b} \mathrm{e}^{\mathrm{i} k r}\right)-\frac{\rho_{a a}}{T_{1}}, \\
& \dot{\tilde{\rho}}_{b a}=\frac{\mathrm{i} \mu E_{0}}{2 \hbar}\left(\rho_{a a}-\rho_{b b}\right) \mathrm{e}^{-\mathrm{i} k r}+\left(\mathrm{i} \Delta-\frac{1}{T_{2}}\right) \tilde{\rho}_{b a}, \\
& \dot{\rho}_{b b}=1-\rho_{a a} .
\end{aligned}
$$

$\Delta$ determines the detuning between the driving frequency $\Omega$ and the molecular resonance frequency $\omega_{0}$, i.e. $\Delta=\left(\omega_{0}-\Omega\right)$. It is assumed in $(6.3-5)$ that the molecular system consists only of two levels $|a\rangle$ and $|b\rangle$. The equations may be modified to describe other situations, e.g. when relaxation to a third level is relevant. In the latter case one has to extend (6.5).

The significance of the relaxation times $T_{1}$ and $T_{2}$ is readily seen, when the electric field is turned off, i.e. for $E_{0}=0$. The energy relaxation time $T_{1}$ describes the relaxation of the population of level $|a\rangle$ with $\rho_{a a}(t)=\rho_{a a}(0) \exp \left(-t / T_{1}\right)$. The phase relaxation time $T_{2}$ determines the decay of the dipole moment $P=$ $N \mu\left(\rho_{b a}+\rho_{a b}\right)$ with $P(t)=P(0) \exp \left(-t / T_{2}\right)$. There are three contributions which determine the dephasing of the system. (i) The macroscopic dipole moment $P$ 
may decay by orientational motion of the individual molecules (orientational relaxation time $T_{\mathrm{R}}$ ); (ii) the molecules may "get out of step", i.e. lose their phase relation by interaction with the surroundings (pure dephasing time $T_{2}^{*}$ ); (iii) the population of the upper level may decay (energy relaxation time $T_{1}$ ). The observed effective dephasing time $T_{2}$ is related to the individual time constants as follows: $1 / T_{2}=1 / T_{\mathrm{R}}+1 / T_{2}^{*}+1 /\left(2 T_{1}\right)$.

The applied electric field excites the system to the upper level $|a\rangle$ via (6.3) and generates a polarization via (6.4). For the following discussion it is important to introduce the pulse area $A=\int \mu E / \hbar d t$ [6.10]. The magnitude of $A$ frequently determines whether a certain nonlinear coherent process occurs or not, e.g. whether a photon echo may be observed. When the applied electric field is strong and the pulse area is large, $A \gg 2 \pi$, repeated excitation and de-excitation processes occur, causing the population $\rho_{b b}$ to oscillate with the Rabi frequency $\chi=\mu E / \hbar$. In most of the following investigations with short pulses one has $A<1$.

Analytical solutions of (6.3-5) can be found for two limiting conditions:

i) Without an applied electric field the density matrix evolves freely. Only the relaxation processes influence $\rho(t)$. The four components of the density matrix may be asigned to a vector $\rho$, as listed in the Appendix [Eq. (6.20)]. The density matrix at time $t$ is obtained by a linear transformation from the initial density matrix $\rho(0)$ :

$$
\rho_{j}(t)=\sum_{k=1}^{4} Y_{j k}(t) \rho_{k}(0) \quad \text { or } \quad \rho(t)=\underline{Y}(t) \rho(0) .
$$

The transformation matrix contains exponentials of $t / T_{2}$ and $t / T_{1}$. It is given explicitly in the Appendix [Eq. (6.21)].

ii) When a resonant electric field of pulse area $A$ is applied, which is short compared to the relaxation times $T_{1}$ and $T_{2}$, the density matrix at the end of the pulse is calculated by a linear transformation $\underline{X}(A)$,

$$
\rho(A)=\underline{X}(A) \rho(0) .
$$

The elements of the transformation matrix $\underline{X}(A)$ depend on the pulse area and the wave vector of the electric field. They are given in the Appendix, [Eq. (6.22)]. The important aspects of many coherent experiments can be seen from the analytical solutions. Experimentally one has periods of short excitations with pulse areas $A_{i}, i=a, b, \ldots$ and free evolution periods of durations $t_{1}, t_{2}, \ldots$ At the time of observation, $t$, after the final excitation pulse with area $A_{\mathrm{f}}$, the density matrix is calculated as a product of the individual excitation and evolution matrices $\underline{X}\left(A_{i}\right), \underline{Y}\left(t_{i}\right)$ :

$$
\boldsymbol{\rho}(t)=\underline{\boldsymbol{Y}}(t) \underline{\boldsymbol{X}}\left(A_{\mathrm{f}}\right) \ldots \underline{\boldsymbol{Y}}\left(t_{2}\right) \underline{\boldsymbol{X}}\left(A_{b}\right) \underline{\boldsymbol{Y}}\left(t_{1}\right) \underline{\boldsymbol{X}}\left(A_{a}\right) \boldsymbol{\rho}(0) .
$$

Detailed examples for the solution of (6.7) are given below in Sect. 6.1.3. Here we refer briefly to the situation depicted in Fig. 6.1c, where three excitation fields are applied. The density matrix at time $t$ is as follows: 


$$
\boldsymbol{\rho}(t)=\underline{\boldsymbol{Y}}(t) \underline{\boldsymbol{X}}\left(A_{c}\right) \underline{\boldsymbol{Y}}\left(t_{2}\right) \underline{\boldsymbol{X}}\left(A_{b}\right) \underline{\boldsymbol{Y}}\left(t_{1}\right) \underline{\boldsymbol{X}}\left(A_{a}\right) \boldsymbol{\rho}(0) .
$$

The treatment given above in $(6.6,7)$ is well justified when the applied light fields are separated in time and the radiating polarization of the sample is small at all times $t_{i}$. In the more general case reference is made to the literature cited in Sect. 6.3.2.

\subsubsection{Ultrafast Coherent Techniques}

A short overview of coherent spectroscopy is given here. Two types of molecular systems are treated: System I contains molecules with a single resonance frequency $\omega_{0}$ (homogenous broadening), while System II shows a distribution of resonance frequencies $f\left(\omega_{0}\right)$ (inhomogeneous broadening).

\section{a) One Excitation Pulse}

In the most elementary coherent experiment one short laser pulse traverses the sample. After the excitation of the system by the pulse with area $A_{a}$ and wave vector $k_{a}$ radiation is emitted from the sample in the direction $k_{a}$. From (6.7) one derives the polarization $P(t)$ observed at the time $t$ after the excitation pulse $P(t)=\mu N\left[\rho_{a b}(t)+\rho_{b a}(t)\right]:$

$$
P(t) \propto \int d \omega f(\omega) \mathrm{e}^{-\mathrm{i} \omega t} \mathrm{e}^{-t / T_{2}} \sin \left(A_{a}\right) \mathrm{e}^{-\mathrm{i} k_{a} r} .
$$

A schematic of the decay of $P(t)$ is shown in Fig. 6.1a. The generated polarization has an amplitude proportional to $\sin \left(A_{a}\right)$; it has the same wave vector as the exciting light pulse. For the molecular system I with $f(\omega)=\delta\left(\omega-\omega_{0}\right)$
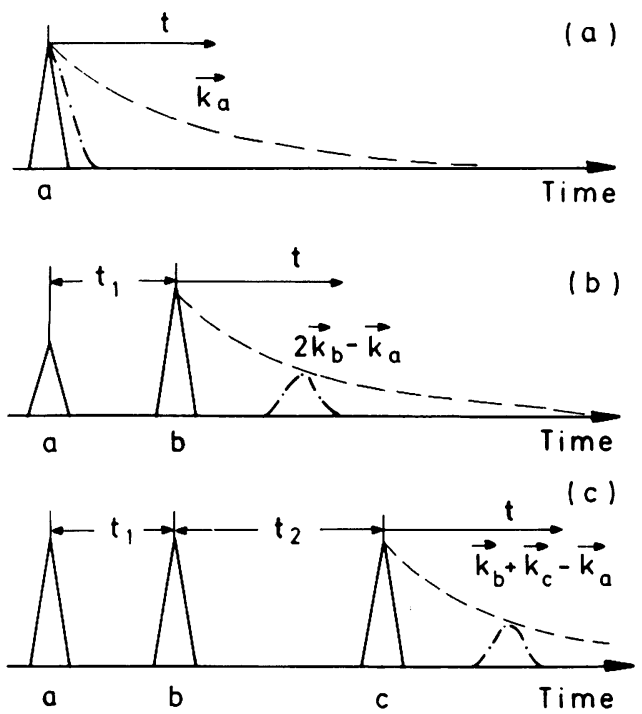

Fig. 6.1a-c. Schematic of various coherent techniques. The electric field pulses (solid triangles) excite the sample. The resulting coherent signal is shown for homogeneously broadened transitions (dashed curves), and for inhomogeneously broadened transitions (dash-dotted curves). (a) A single excitation pulse with wave vector $k_{\mathrm{a}}$. Homogeneously broadened transitions lead to an exponential decay with dephasing time $T_{2}$, while inhomogeneous broadening induces a faster decay. (b) Two excitation pulses a and b with wave vectors $\boldsymbol{k}_{\mathrm{a}}$ and $\boldsymbol{k}_{\mathrm{b}}$ give echos in the direction $2 \boldsymbol{k}_{\mathrm{b}}-\boldsymbol{k}_{\mathrm{a}}$. For inhomogeneously broadened transitions the echo is emitted at time $t=t_{1}$. The echo amplitude measured as a function of $t_{1}$ allows determination of the dephasing time $T_{2}$. (c) When three excitation pulses are used the coherent signal emitted in the direction $\boldsymbol{k}_{\mathrm{b}}+\boldsymbol{k}_{\mathrm{c}}-\boldsymbol{k}_{\mathrm{a}}$ allows determination of the dephasing time $T_{2}$ and the energy relaxation time $T_{1}$ 
(broken line in Fig. 6.1a), the polarization decays exponentially with the dephasing time $T_{2}$. For a distribution of resonance frequencies $f\left(\omega_{0}\right)$ the decay of polarization starts exponentially, but accelerates due to the interference of the different resonance frequencies (dash-dotted line in Fig. 6.1a). With increasing width of the frequency distribution, the decay becomes more rapid and it is difficult - often impossible - to determine the dephasing time $T_{2}$. The explicit mathematical solution for the signal decay is readily obtained from (6.8). We note that the polarization is proportional to the product of the Fourier transform $g(t)$ of the distribution function $f\left(\omega_{0}\right)$ of the resonance frequencies times the exponential decay with $\exp \left(-t / T_{2}\right)$.

Investigations with single-pulse excitation are well established in ultrafast coherent spectroscopy. A series of interesting experiments are discussed in Sect. 6.2.

\section{b) Two Excitation Pulses}

When two excitation pulses of areas $A_{a}$ and $A_{b}$ separated by the evolution time $t_{1}$ (see Fig. 6.1b) are applied to the (molecular) system, a more complex time dependence of the coherent signal or of the density matrix is found. At time $t$ after the second pulse, the radiating polarization consists of three contributions: two parts are independently produced by the two exciting pulses, radiating in the corresponding directions $k_{a}$ and $k_{b}$. The two polarizations are described by (6.8). Of special interest is a third term, where the polarization evolves as follows:

$$
\begin{aligned}
P_{2 \mathrm{PE}}(t) \propto \int & \int \omega(\omega) \exp \left[-\mathrm{i} \omega\left(t-t_{1}\right)-\frac{t+t_{1}}{T_{2}}\right] \\
& \times \sin A_{a} \sin ^{2} \frac{A_{b}}{2} \exp \left[-\mathrm{i}\left(2 k_{b}-k_{a}\right) r\right] .
\end{aligned}
$$

The signal is emitted with the wave vector $2 k_{b}-k_{a}$ and may readily be separated from the exciting beams. For a homogeneously broadened transition (System I), the peak of the polarization is proportional to $\exp \left(-t_{1} / T_{2}\right)$. The polarization decays at later times with $\exp \left(t / T_{2}\right)$ (see Fig. 6.1b, broken line). Quite different is the situation for strong inhomogeneous broadening (System II). Now the (underlined) interference term in (6.9) is removed at one delay time, $t=t_{1}$, and a delayed signal appears, i.e. one finds a photon echo with an amplitude proportional to $\exp \left(-2 t_{1} / T_{2}\right)$ [6.11-13]. Variation of $t_{1}$, i.e. of the distance between the two exciting pulses, therefore enables one to deduce the dephasing time $T_{2}$ even for inhomogeneously broadened transitions [6.13]. (For the intermediate case with small inhomogeneous broadening see [6.14]).

An important aspect for the application of the photon echo technique is the dependence of the signal on the area of the excitation pulses. Good echo signals are generated for $A_{a} \simeq \pi / 2$ and $A_{b} \simeq \pi$. For small areas $A_{a}, A_{b} \ll \pi$ the signal 
amplitude drops with $A_{a} A_{b}^{2}$ or the signal intensity with $\left(A_{a} A_{b}^{2}\right)^{2}$. We recall that in the case of a single excitation pulse the emitted signal intensity is proportional to $A_{a}^{2}$. Consequently, coherent echo experiments become more difficult when the transitions are weak and the pulses and relaxation times are very short.

\section{c) Three Excitation Pulses}

When three excitation pulses with areas $A_{a}, A_{b}$, and $A_{c}$ separated in time by $t_{1}$ and $t_{2}$ are applied to the molecular system, a variety of coherent signals is produced: single excitation signals, two-pulse echos from each pair of the three pulses, and so-called three-pulse echos at the time $t=t_{1}$ after the third pulse, the latter echos are emitted in the direction $k_{b}+k_{c}-k_{a}$ (see Fig. 6.1c). The introduction of the third pulse allows one to measure the dephasing time $T_{2}$ of the molecular transition and to determine the energy relaxation time $T_{1}$. The application of three pulses gives considerable flexibility to the design of experiments. Similar limitations regarding the signal strength exist as in two-pulse experiments; the three-pulse echo intensities are proportional to $\left(\sin A_{a} \sin A_{b} \sin A_{c}\right)^{2}$. For small area pulse the echo signal decreases proportion to $A_{1}^{2} A_{2}^{2} A_{3}^{2}$. Experiments with ultrafast three-pulse echos have been performed for a number of electronic transitions. These are discussed in Sect. 6.3.2.

\subsubsection{Electric Dipole and Raman-Type Transitions}

The interaction between the system of interest and the light field depends on the specific type of interaction Hamiltonian $H_{\mathrm{ie}}$. We address here two situations, the electric dipole and the Raman-type transitions. The common aspect of the (molecular) excitation is best demonstrated by introducing the operator $\hat{q}$ for the excitation coordinate (of the molecules). In the case of a vibrational transition, $\hat{q}$ represents the coordinate of the nuclear motion of the vibrating molecules. For an electronic transition, $\hat{q}$ is related to the motion of the electrons. For an ensemble of two-level systems the expectation value $\langle\hat{q}\rangle$, called the coherent or collective amplitude, is given by:

$$
\langle\hat{q}\rangle=\operatorname{Tr}\{\rho \hat{q}\}=q\left(\rho_{a b}+\rho_{b a}\right),
$$

where $q_{a b}=q=\langle a|\hat{q}| b\rangle$ is the transition matrix element.

\section{a) Electric Dipole Transitions}

Electric dipole transitions are commonly investigated with light frequencies close to a resonance frequency. In this case the interaction cross sections are large and the polarizations resulting from the excitation process generate new electromagnetic waves.

The electric dipole moment $\mu$ related to the transition between the levels $|a\rangle$ and $|b\rangle$ (of one molecule) is a vector; its direction (unit vector $\boldsymbol{u}$ ) is fixed in 
the reference frame of the molecule and its amplitude is proportional to the amplitude of the coordinate $q: \boldsymbol{\mu}=\boldsymbol{u} d \mu / d q q[6.15,16]$. The interaction of the dipole moment with an electric field is given by the Hamiltonian $H_{i e}=-\boldsymbol{\mu} \boldsymbol{E}=$ $d \mu / d q q E \cos \theta$, where $\theta$ is the angle between the dipole moment and the electric field. For an ensemble of molecules the relaxation of the total dipole moment $\langle\boldsymbol{\mu}\rangle$ as a function of time depends on (i) the orientational relaxation of the vector $\boldsymbol{u}$ (irrelevant in most solids) and (ii) the relaxation of the absolute value of the coherent amplitude $\langle\hat{q}\rangle$. This topic is the subject of intensive investigations discussed in this chapter.

It is of interest to compare the quantities measured in time-resolved coherent investigations and in steady-state spontaneous spectroscopy, i.e. the time dependence of the expectation values $\langle\hat{q}\rangle$ or $\langle\boldsymbol{\mu}\rangle$ with the absorption spectra $[6.3,15]$. The latter can be calculated from $(6.3-5)$, when the left-hand side of the equations, i.e. the time-dependent terms, are equal to zero. One readily finds the absorption line to be of Lorentzian shape with a peak absorption cross section $\sigma=(d \mu / d q)^{2} \omega T_{2} /\left(\varepsilon_{0} c n \hbar\right)$ and a linewidth (FWHM) of $\Delta v=1 / \pi T_{2}$. Here $\varepsilon_{0}$ is the permittivity, $n$ the refractive index, $c$ the velocity of light, and $\hbar$ Planck's constant.

The absorption spectrum $J(\omega)$ may be related to the time-dependent dipole moment in a general way without referring to the special model introduced above. Line-shape theory gives $[6.2-6,15,16]$ :

$$
J(\omega) \propto \int_{-\infty}^{+\infty} \mathrm{e}^{\mathrm{i} \omega t} \phi(t) d t=\int_{-\infty}^{+\infty} \mathrm{e}^{\mathrm{i} \omega t}\langle\langle\mu(t) \mu(0)\rangle\rangle d t,
$$

where $\langle\langle\cdots\rangle\rangle$ denotes the equilibrium ensemble average. According to (6.11) the spectral line shape is the Fourier transform of the correlation function $\phi(t)$, i.e. of the time dependence of $\mu(t)$, which is measured in straightforward coherent experiments [see Sect. 6.1.3(a)]. Introducing an exponential decay with time constant $T_{2}$ for the dipole moment in (6.11) one obtains the same Lorentzian line shape as determined above directly from (6.3-5).

\section{b) Raman-Type Transitions}

Raman type transitions have the advantage that excitation is possible with a wide range of available electromagnetic fields, i.e. resonance frequencies are not required. On the other hand, the Raman interaction is a weak, second-order process; the pulse area and the degree of excitation are small. In spite of this difficulty, ultrafast Raman spectroscopy has provided valuable new information. Direct observation of several rapid dynamic processes has become possible and superior spectral resolution was demonstrated in congested spectral regions.

According to Placzek [6.17], the interaction between a given vibrational mode and the electric field can be described by the interaction Hamiltonian $H_{\mathrm{ie}}=(\partial \underline{\alpha} / \partial q) q E E / 2$, where $d \underline{\alpha} / d q$ stands for the change of the electric polarizability tensor with the vibrational coordinate $q$. It leads to the excitation of the molecular mode (frequency $\omega_{0}$ ) under the action of the light field $E$, provided 
that components $i, j$ of the electric field exist, where the product $E_{i} E_{j}^{*}$ oscillates at the frequency $\omega_{0}$. The Raman process may again be described by (6.3-5), if we replace the quantity $\mu E_{0}$ by $(\partial \underline{\alpha} / \partial q) q_{a b} E_{i} E_{j} / 2$. In a similar way, the radiating polarization is obtained as

$$
P_{i}=N \mu_{i}=N \frac{\partial \underline{\alpha}_{i j}}{\partial q} E_{j}\langle\hat{q}\rangle
$$

The polarization is proportional to the electric field $\boldsymbol{E}$, i.e. the molecular excitation $\langle q\rangle$ leads to a scattered light field when monitored by a probing field. Without a probing field the coherent amplitude $\langle\hat{q}\rangle$ does not produce electromagnetic radiation. When the transition is electric dipole and Raman active, some light emission may occur, provided the molecules are in a crystal without inversion symmetry. This case will not be treated here. For isotropic Raman transitions the tensor $\partial \underline{\alpha} / \partial q$ is reduced to a scalar quantity $\partial \alpha / \partial q$. In this case, rotational motion of the molecules does not effect the evolution of the coherent signal and the decay of the coherent amplitude $\langle q\rangle$. The more complex case of an anisotropic Raman tensor has been treated in the literature by Laubereau and Kaiser [6.7], by Kohles and Laubereau [6.18], and by Dick [6.19]; it will not be discussed here in detail.

Comparing time-resolved coherent and spontaneous Raman scattering, one obtains very similar equations as in the case of electric dipole transitions. The spontaneous Raman line shape can again be calculated from (6.3-5) or from the line-shape theory [6.2-6]. The spectral shape is the Fourier transform of a molecular correlation function; for isotropic Raman lines it is proportional to the Fourier transform of $\langle\langle q(t) q(0)\rangle\rangle$.

\section{c) Pulse Area and Population Changes in Ultrafast Coherent Experiments}

For practical experiments it is important to know how strongly the (molecular) system is excited under the action of a light pulse. The strength of the excitation determines which type of coherent experiment can be performed with given light pulses. The important quantity is the pulse area $A$ which is directly related to the changes of the population of the various levels [see $(6.6,22)]$; e.g. for a system initially in the ground state a short pulse with area $A$ changes the ground-state population by $\Delta \rho_{b b}=(1-\cos A) / 2$. Using an exciting pulse with peak intensity $I$, duration $t_{\mathrm{p}}$, and absorption cross section $\sigma$ one estimates the pulse area to be $A=\mu E_{0} t_{\mathrm{p}} / \hbar=\left(2 n^{2} I T_{2} \sigma / \hbar \omega_{0}\right)^{1 / 2}$. We assume here that the duration of the pulses is equal to the dephasing time. We now give numbers for the pulse area for three relevant examples using the following parameters: dephasing time $T_{2}=10^{-12} \mathrm{~s}$, intensity $I=10^{10} \mathrm{~W} / \mathrm{cm}^{2}$, refractive index $n=1.5$.

i) For an infrared-active vibrational $\mathrm{CH}$-stretching mode at $\lambda=3 \mu \mathrm{m}$ with $\sigma=10^{-19} \mathrm{~cm}^{2}$ one obtains $A=2.6 \times 10^{-1}$ and $\Delta \rho_{b b}=1.6 \times 10^{-2}$.

ii) For an electronic transition at $\lambda=0.5 \mu \mathrm{m}$ with $\sigma=10^{-16} \mathrm{~cm}^{2}$ one calculates $A=3.4$ or $\Delta \rho_{b b}=0.99$. The two examples show that electronic transi- 
tions may lead to large pulse areas making coherent experiments quite easy, while for vibrational transitions the observation of two- and three-pulse echos with echo amplitudes proportional to $A^{6}$ is difficult.

iii) For Raman active transitions the pulse area $A=1 / 2(\partial \alpha / \partial q) q_{a b} t_{\mathrm{p}} E^{2} / \hbar$ may be calculated using the definitions of $\partial \alpha / \partial q$ and $q_{a b}$ from the literature [6.8]. One obtains $A=\operatorname{In} \lambda^{2} t_{\mathrm{p}} \sqrt{\delta \sigma} /(\pi c \hbar)$, where $\delta \sigma$ is the Raman cross section integrated over one Steradian. Taking $\delta \sigma=2.5 \times 10^{-29}$ from benzene [6.10] (which has a rather large Raman cross section for a molecular vibration) and $\lambda=0.5 \mu \mathrm{m}$ we compute an area $A$ of $A=1.9 \times 10^{-2}$ or a population change $\Delta \rho_{b b} \simeq 10^{-4}$. These numbers are even smaller than the values calculated for resonant infrared absorption, see (i). It is evident that under the present conditions, ultrafast Raman-echo experiments appear to be unrealistic, while single-pulse coherent Raman experiments are readily possible. It should be noted that considerably higher cross sections are found for resonant Raman transitions. Only under these conditions might ultrafast Raman-echo experiments become possible.

In this section a set of equations was introduced which allows one to treat time-resolved coherent spectroscopy within the framework of the model presented in Sect. 6.1.1. We have briefly outlined and compared different experimental methods for which applications are discussed in the following sections.

\subsection{Coherent Spectroscopy Using a Single Excitation Process}

We focus here on investigations of time-resolved coherent spectroscopy using single excitation processes. It is the aim of this section to demonstrate the different time-resolved techniques and to show the advantages of coherent techniques over spontaneous frequency-domain methods.

\subsubsection{Time-Resolved Coherent Raman Spectroscopy of a Single Homogeneously Broadened Transition}

The experiments of time-resolved coherent Raman spectroscopy are performed in two steps. First, the sample is excited by a pair of light pulses via transient stimulated Raman scattering (see Fig. 6.2, upper part) [6.20-23]. Subsequently, the degree of excitation is interrogated by a probe pulse, which is delayed in time relative to the excitation pulses. The probe pulse monitors the coherent amplitude by Raman scattering $[6.1,7,8]$. The experiments are described by two different sets of equations: the material equation governing the generation of the excitation under the action of the light fields [see (6.3-5)] and the wave equation (6.2) determining the light field due to the interaction with the material excitation. We recall that in coherent Raman processes the pulse areas are quite small [see Sect. 6.1.4(c)]. For this reason the changes of the diagonal elements of the density matrix are small and the off-diagonal elements may be calculated by using only 
Excitation
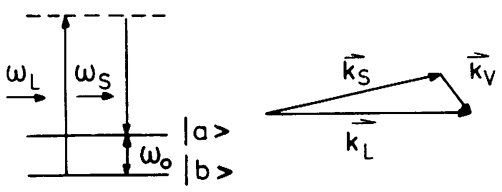

Anti-Stokes-Probing
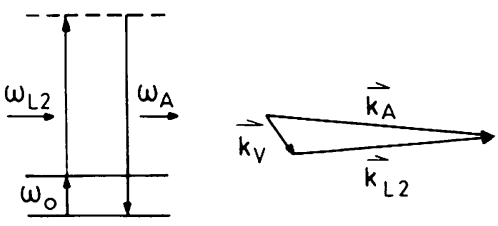

Fig. 6.2. Schematic of a time-resolved coherent Raman experiment. The excitation of the upper level $|a\rangle$ is accomplished via stimulated Raman scattering by the laser and Stokes pulses at frequencies $\omega_{\mathrm{L}}$ and $\omega_{\mathrm{s}}$, respectively. The wave vectors of the two laser fields determine the wave vector of the coherent excitation, $\boldsymbol{k}_{\mathrm{v}}=\boldsymbol{k}_{\mathrm{L}}-\boldsymbol{k}_{\mathrm{s}}$ (upper part). At a later time the coherent Raman probing process (lower part) with a second laser pulse $E_{\mathrm{L} 2}$ $\left(\omega_{\mathrm{L} 2}, \boldsymbol{k}_{\mathrm{L} 2}\right)$ monitors the coherent excitation. The coherent anti-Stokes signal emitted under phase matching conditions $\boldsymbol{k}_{\mathrm{a}}=\boldsymbol{k}_{\mathrm{L} 2}-\boldsymbol{k}_{\mathrm{v}}$ is a measure of the coherent amplitude at the probing time

(6.4). For Raman-type interactions (6.4) may be solved by direct integration. The electric field producing excitation consists of two components:

$$
E=E_{\mathrm{L}} \cos \left(-\omega_{\mathrm{L}} t+k_{\mathrm{L}} x\right)+E_{\mathrm{s}} \cos \left(-\omega_{\mathrm{s}} t+k_{\mathrm{s}} x\right),
$$

and for the coherent material excitation one makes the ansatz of a plane wave

$$
\langle q\rangle=\frac{\mathrm{i}}{2} Q \exp \left(-\mathrm{i} \omega_{0} t+k_{\mathrm{v}} x\right)+\text { c.c. }=q_{a b}\left(\rho_{a b}+\rho_{b a}\right) .
$$

With these expressions (6.4) yields the amplitude $Q$ after one integration $[6.1,7,8,24]$ :

$$
Q(x, t)=\frac{\mathrm{i} q_{a b}^{2}}{2 \hbar} \frac{\partial \alpha}{\partial q} \int_{-\infty}^{t} E_{\mathbf{L}}\left(t^{\prime}, x\right) E_{\mathrm{s}}^{*}\left(t^{\prime}, x\right) \exp \left[\frac{1}{T_{2}}\left(t^{\prime}-t\right)+\mathrm{i} \Delta t^{\prime}\right] d t^{\prime} .
$$

The excitation process is shown schematically in Fig. 6.2, upper part. The two components of the electric field at frequency $\omega_{\mathrm{L}}$ and $\omega_{\mathrm{s}}$ drive the vibration with frequency $\Omega=\omega_{\mathrm{L}}-\omega_{\mathrm{s}}$ close to the resonance frequency $\omega_{0}$. The wave vector of the coherent amplitude $k_{\mathrm{v}}$ is determined by $k_{\mathrm{v}}=k_{\mathrm{L}}-k_{\mathrm{s}} . Q$ shows the properties discussed previously: a rapid rise with the driving force $E_{\mathrm{L}} E_{\mathrm{s}}^{*}$ and an exponential decay with $\exp \left(-t / T_{2}\right)$ of the freely vibrating system. The 'back' reaction of the coherent amplitude on the light fields is governed by the polarization $\boldsymbol{P}$ :

$$
\boldsymbol{P}=N \frac{\partial \alpha}{\partial q}\langle q\rangle \boldsymbol{E}+\underline{\chi}_{\mathrm{NR}}^{(3)} \varepsilon_{0} \boldsymbol{E E E}
$$

The coupling of the wave equation and the material equation via the polarization leads to stimulated Raman scattering or Stokes/anti-Stokes coupling, which 
have been discussed in detail elsewhere [6.8,20-23,25-28]. The nonresonant nonlinear polarization $\chi_{\mathrm{NR}}^{(3)}$ is due to distant electronic transitions. It leads to self-phase-modulation and nonresonant scattering $[6.10,28]$.

For time-resolved coherent Raman spectroscopy a probing pulse is necessary to obtain information on the time dependence of the coherent amplitude (see Fig. 6.2, lower part). The third light pulse $E_{\mathrm{L} 2}=E_{\mathrm{L} 2}(t) \cos \left(-\omega_{\mathrm{L} 2} t+k_{\mathrm{L} 2} x\right)$ generates a radiative polarization according to (6.14). A short probing pulse at variable time delay $t_{\mathrm{D}}$ after the excitation pulses allows one to map out the time dependence of the coherent amplitude. The scattered signal at the anti-Stokes frequency $\omega_{\mathbf{A}}=\omega_{\mathbf{L} 2}+\omega_{0}$ or at the Stokes frequency $\omega_{\mathrm{s} 2}=\omega_{\mathrm{L} 2}-\omega_{0}$ is observed. Efficient generation of the coherent signal requires phase matching, i.e. the wave vectors of the material excitation $k_{\mathrm{v}}$, the probing field $\boldsymbol{k}_{\mathrm{L} 2}$, and generated light, e.g. $\boldsymbol{k}_{\mathrm{A}}$, have to satisfy the condition $\boldsymbol{k}_{\mathrm{v}}+\boldsymbol{k}_{\mathrm{L} 2}=\boldsymbol{k}_{\mathrm{A}}$.

\section{a) Experimental Arrangements}

A typical experimental system is depicted schematically in Fig. 6.3. A pumping laser source generates exciting and probing light fields at the frequencies $\omega_{\mathrm{L}}, \omega_{\mathrm{s}}$, and $\omega_{\mathrm{L} 2}$ in several frequency converters. In early experiments a simple optical system was used: the Stokes pulse was generated via stimulated Raman scattering of the strong laser pulse in the sample itself $[6.29,30]$. More recently, frequency conversion by dye lasers produced tunable Stokes pulses [6.31-34]. The exciting

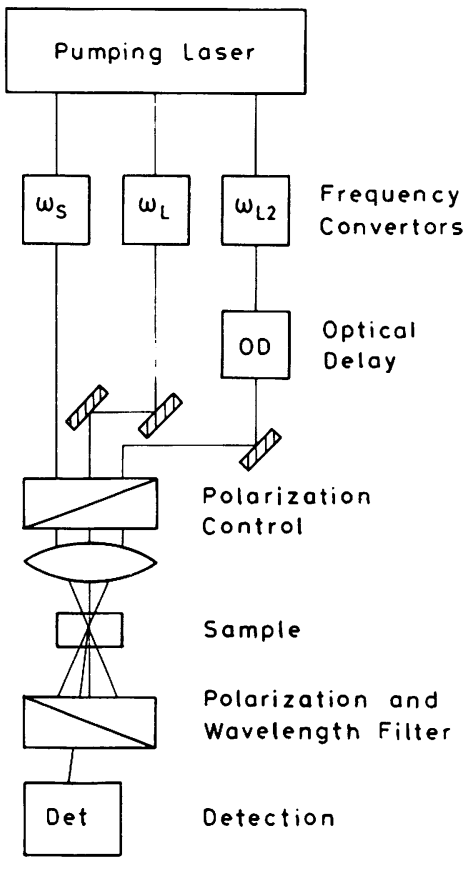

Fig. 6.3. Experimental system used for time-resolved coherent Raman scattering. Synchronized pulses at frequencies $\omega_{\mathrm{L}}, \omega_{\mathrm{s}}$, and $\omega_{\mathrm{L} 2}$ are generated by different frequency converters from one pumping laser source. The pulses at $\omega_{\mathbf{L}}$ and $\omega_{\mathrm{s}}$ excite the sample via stimulated Raman scattering. The coherent excitation is monitored by the delayed (optical delay OD) probing pulses at frequency $\omega_{\mathrm{L} 2}$. The different beams cross in the sample at phase-matching angles. Polarization and wavelength filters separate the coherent signal from the excitation light 
Stokes and laser pulses are focused at a specific angle into the sample generating the coherent material excitation. The probing light pulse at frequency $\omega_{\mathrm{L} 2}$ is delayed relative to the excitation process in the optical delay system OD and monitors the excited volume of the sample. The geometry of the exciting and probing beams in the sample have to be adjusted to obtain phase matching for the coherent probe-scattering process. In most experiments the signal intensities are much smaller than the intensities of the excitation pulses. Consequently, the coherently scattered light must be separated carefully from the incoming light beams by diaphragms and spectral and/or polarization filtering. The detection system is a highly sensitive photo-detector. Frequently, the spectra of the coherently scattered light are analyzed with the help of a spectrograph.

\section{b) Dephasing in Liquids}

A straightforward application of time-resolved coherent Raman spectroscopy on the picosecond time scale is the investigation of single Raman active vibrational transitions. Such an experiment was performed for the first time by von der Linde et al. [6.29] in 1971. The authors observed the coherent anti-Stokes signal for ethanol $\left(\mathrm{C}_{2} \mathrm{H}_{5} \mathrm{OH}\right)$ and carbontetrachloride $\left(\mathrm{CCl}_{4}\right)$ on the picosecond time scale.

A large number of other molecules and transitions have been studied in the meantime [6.32-43]. The state of the art of coherent measurements with high time resolution is demonstrated in two recent publications by Gale et al. [6.42] and by Zinth et al. [6.44]. In the first experiment, a Nd: glass laser system supplied light pulses with relatively long duration $\left(t_{\mathrm{p}}=5 \mathrm{ps}\right)$. The high time resolution was achieved by a specially designed excitation and probing system that allows the signal decay to be measured over 9 orders of magnitude. With the nearly Gaussian-shaped pulses from the $\mathrm{Nd}$ :glass laser, the decay of the response function (see broken curve in Fig. 6.4) accelerates with decreasing signal at later delay times. As a consequence, the favorably high signal-to-noise ratio of the experiment allowed the measurement of decay times considerably shorter than the pulse duration. In Fig. 6.4a the solid curve corresponding to the $v_{1}$ mode of acetonitrile at $v / c=2943 \mathrm{~cm}^{-1}$ shows an exponential decay over the entire observation range. This fact clearly demonstrates that the vibrational transition is homogeneously broadened with a dephasing time $T_{2}=1.63 \pm 0.07 \mathrm{ps}$.

Superior time resolution is achieved with very short light pulses. $Z$ inth et al. [6.44] introduced an experimental system with femtosecond pulses $\left(t_{\mathrm{p}} \simeq 80 \mathrm{fs}\right)$ from a ring dye laser. A recent result is presented in Figs. $6.4 \mathrm{~b}$ and 6.5. In Fig. $6.4 \mathrm{~b}$ the same acetonitrile mode was studied as in Fig. 6.4a. Using femtosecond pulses the coherent signal is well separated from the autocorrelation trace, just $100 \mathrm{fs}$ after time zero. Consequently, the decay time is determined with very high precision to be $T_{2}=1.70 \pm 0.02$ ps. In Fig. $6.5 \mathrm{a}$ and $\mathrm{b}$ the coherent signal decays are shown for the $v_{2}$ and $v_{7}$ modes of liquid acetone. In both cases one finds for delay times larger than $200 \mathrm{fs}$ a clear exponential decay. The time constants are $510 \pm 30 \mathrm{fs}\left(v_{7}\right.$ mode) and $305 \pm 10 \mathrm{fs}\left(v_{2}\right.$ mode); i.e. one obtains 

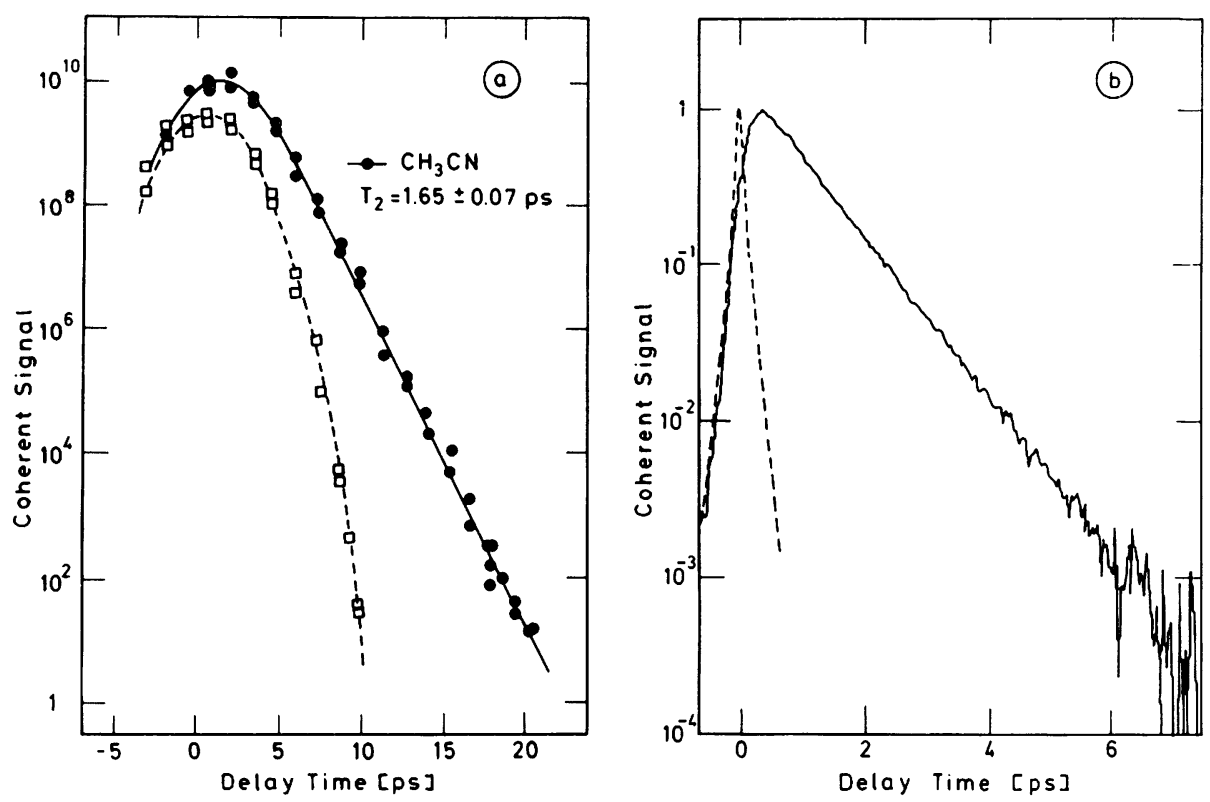

Fig. 6.4a, b. Coherent anti-Stokes signal for the $v_{1}$ mode of acetonitrile plotted as a function of the time delay of the probing pulses. (a) Laser pulses of 5 ps duration were used. The signal is recorded over nine orders of magnitude. $\bullet$ : Coherent response of acetonitrile $\mathrm{CH}_{3} \mathrm{CN}$. From the exponential decay a dephasing time $T_{2}=1.65 \pm 0.07 \mathrm{ps}$ is determined. $\circ$ : Response function of the experimental system measured by coherent scattering in $\mathrm{C}_{2} \mathrm{H}_{5} \mathrm{OH}$. (Redrawn from Gale et al. [6.42]). (b) Laser pulses of 80 fs duration were used. A ready separation between coherent signal and autocorrelation trace starts within $100 \mathrm{fs}$. The dephasing time is determined to be $T_{2}=1.70 \pm 0.02 \mathrm{ps}$ [6.44b]

dephasing times of $T_{2}=1020 \pm 60 \mathrm{fs}$ and $610 \pm 20 \mathrm{fs}$. At shorter delay times the signal deviates from the pure exponential decay. In Fig. 6.5a this effect is mainly due to the nonresonant susceptibility. There may also be some residual influence from collisional processes (see below) which are not included in (6.3-5).

The experiments on time-resolved coherent Raman scattering have initiated a number of theoretical investigations treating dephasing and energy relaxation of vibrational levels in molecular liquids. Fischer and Laubereau [6.2] have estimated relaxation times assuming a binary collision between the molecules with exponential intermolecular interaction potential. Despite its several simplifying assumptions this model is capable of predicting dephasing times in liquids over a range of parameters such as temperature, concentration, and viscosity. A number of other theoretical approaches, e.g. molecular dynamics simulations, correlation function modelling, or hydrodynamic models have been discussed in the literature [6.45-60]. For a review see Oxtoby [6.3].

In many coherent experiments the initial signal is strongly affected by the nonresonant susceptibility $\chi_{\mathrm{NR}}^{(3)}$ deviating substantially from the single exponential decay discussed theoretically (for an example see Fig. 6.5 or 6.6). The influence 


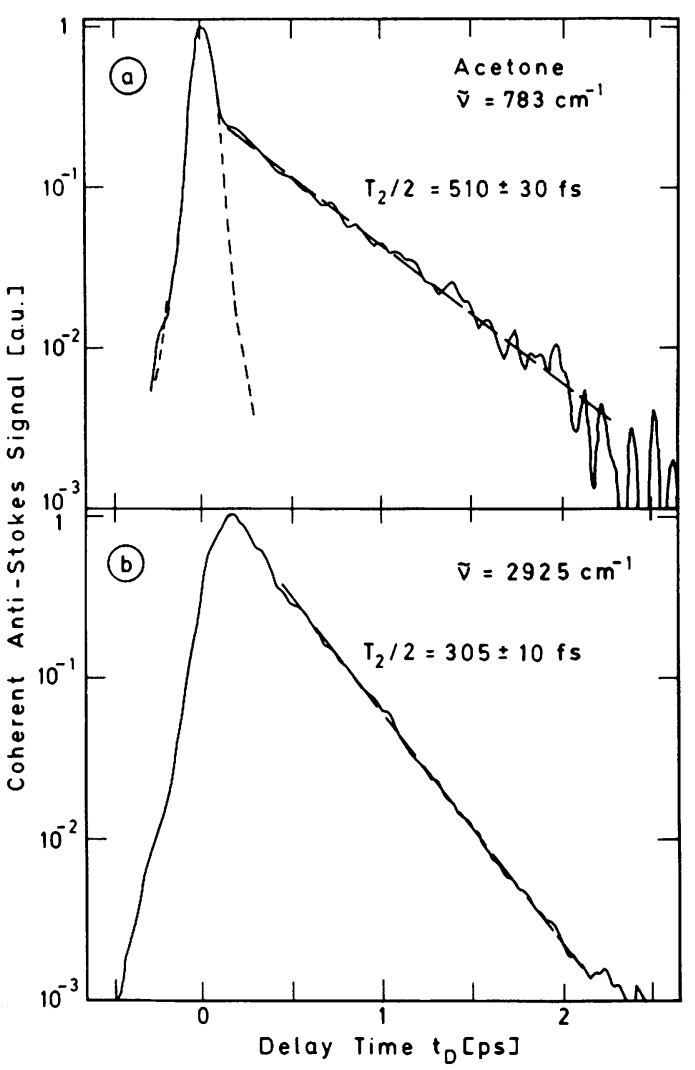

Fig. 6.5a, b. Coherent signal from aceton measured with femtosecond laser pulses. The $v_{7}(\mathbf{a})$ and $v_{2}$ (b) modes are studied. Exponential decay with time constants $T_{2} / 2=510 \pm 30 \mathrm{fs}\left(v_{7}\right)$ and $305 \pm 10 \mathrm{fs}$ $\left(v_{2}\right)$ are found at later delay times. The signal shape in (a) around time zero is influenced by the nonresonant susceptibility $\chi_{\mathrm{NR}}^{(3)}$. The dashed curve shows the response function of the experiment measured with the help of sum-frequency generation in a nonlinear KDP crystal. The narrow response function clearly demonstrates the power of the experimental system to measure very short decay times [6.44]

of the nonresonant susceptibility $\chi_{\mathrm{NR}}^{(3)}$ on time-domain coherent Raman experiments was investigated for the first time by Zinth et al. [6.61] in mixtures, where molecular modes of highly diluted molecules were studied. Figure 6.6 shows the data for the $v_{1}$ mode of liquid $\mathrm{CCl}_{4}\left(9\right.$ vol.\%) dissolved in cyclohexane, $\mathrm{C}_{6} \mathrm{H}_{12}$, (experimental system $\mathrm{Nd}$ :glass laser with $t_{\mathrm{p}}=7 \mathrm{ps}$ ). The peak of the scattered Stokes signal is close to $t_{\mathrm{D}}=0$, where the driving force $\left(E_{\mathrm{L}} E_{\mathrm{s}}^{*}\right)$ overlaps with the probing light pulse. At first the signal decays very rapidly by a factor of 50 ; it subsequently decays more slowly with a time constant of $T_{2} / 2=3.6 \mathrm{ps}$ (broken line). This time dependence is readily understood by taking into account the nonresonant susceptibility $\chi_{\mathrm{NR}}^{(3)}$ of the system [see (6.14)]. The nonresonant susceptibility induces an additional signal close to time zero due to a polarization proportional to $\chi_{\mathrm{NR}}^{(3)} E_{\mathrm{L}} E_{\mathrm{s}} E_{\mathrm{L} 2}$. This part of the signal has the time dependence of the experimental response function. At later delay times, the slower decay due to the resonantly excited solute molecules $\left(\mathrm{CCl}_{4}\right)$ takes over and determines the decay curve. Under the existing experimental conditions the later decay is close to an exponential (further details are given together with the discussion of results on pure $\mathrm{CCl}_{4}$ in Sect. 6.2.2). Comparing the experimental signal curves with the 


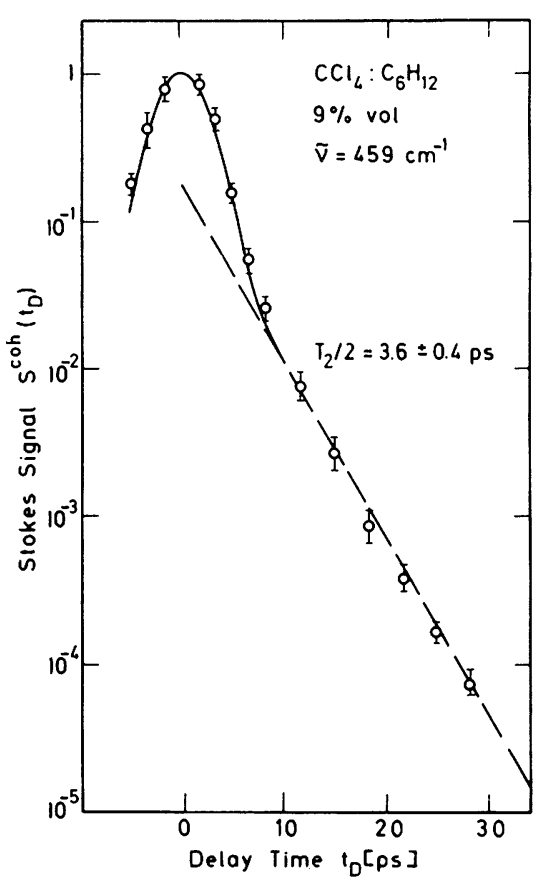

Fig. 6.6. Coherent signal from a mixture of $\mathrm{CCl}_{4}$ and $\mathrm{C}_{6} \mathrm{H}_{12}$. Here, the effect of the nonresonant susceptibility $\chi_{\mathrm{NR}}^{(3)}$ in a time resolved Raman experiments was demonstrated for the first time. While the exponential decay at later delay times is due to the resonant coherent excitation of the $\mathrm{CCl}_{4}$ molecules, the signal peak at time zero originates from the nonresonant susceptibility $\chi_{\mathrm{NR}}^{(3)}$ of the solvent molecules $\mathrm{C}_{6} \mathrm{H}_{12}$ [6.61]

theory of $(6.3-5)$ and $(6.13,14)$ allows one to deduce the ratio of the nonresonant to the resonant susceptibility in the mixture.

The experimental results show that the resonant and the nonresonant part of the nonlinear susceptibility may be separated in a time-domain experiment. While at time zero, both the nonresonant and the resonant part contribute to the signal, the measurement at later delay time gives access to the resonant part of the susceptibility. This separation allows frequency-domain coherent antiStokes Raman experiments (CARS) without the disturbing influences of $\chi_{\mathrm{NR}}^{(3)}$; picosecond light pulses and delayed probing with tuning of the frequency difference $\omega_{\mathrm{L}}-\omega_{\mathrm{s}}$ are required (see [6.61-64]).

In the experiment of Fig. 6.6 the strong Raman band of $\mathrm{CCl}_{4}$ at $\tilde{v}=459 \mathrm{~cm}^{-1}$ was investigated and the influence of the nonresonant susceptibility of the solvent cyclohexane was evident because of the high dilution of the $\mathrm{CCl}_{4}$ molecules, which reduced the resonant contribution to the signal. There are many experiments, where $\chi_{\mathrm{NR}}^{(3)}$ contributes to the probe signal even in concentrated samples. This situation occurs, when (i) weak Raman transitions are investigated, (ii) very strong nonresonant susceptibilities exist, as e.g. in semiconductors, or (iii) the excitation process is very transient, i.e. when the duration of the excitation pulses is much shorter than the dephasing time $T_{2}$. The latter case was realized under the experimental conditions of Fig. 6.5a, where the excitation pulses of $t_{\mathrm{p}}=70 \mathrm{fs}$ were considerably shorter than the dephasing time $T_{2}=1 \mathrm{ps}$. 


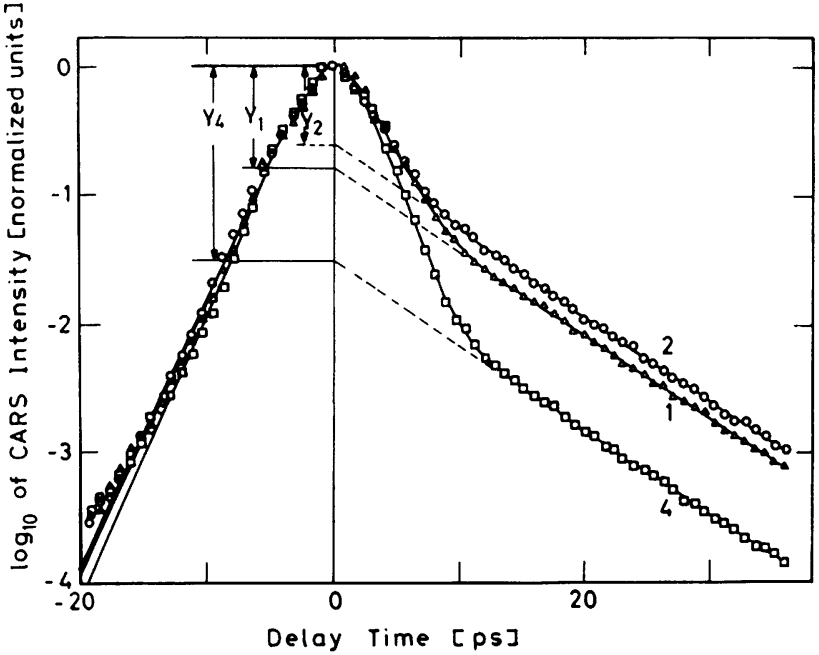

Fig. 6.7. Time-resolved CARS signal for LO-phonons in $\mathrm{GaP}$ measured for different polarization conditions showing the influence of the nonresonant susceptibility $\chi_{\mathrm{NR}}^{(3)}$ (around time zero) and the exponential decay of the resonant signal at later delay times [6.65]

An interesting example, where a time-domain experiment allows determination of the components of the nonresonant susceptibility tensor $\chi_{\mathrm{NR}}^{(3)}$ in crystalline gallium phosphate, GaP, is presented in Fig. 6.7 [6.65]. The coherent anti-Stokes signal was recorded for three different polarization conditions of excitation and probing fields (curves 1,2, and 4 in Fig. 6.7). The slow exponential decay at later times corresponds to the LO-Raman resonance of $\mathrm{GaP}$ at $403 \mathrm{~cm}^{-1}$. Each configuration of polarization of excitation and probing light fields yields the same dephasing time for the LO-phonons, but a different ratio between nonresonant and resonant part of the signal (represented by the values $Y_{i}$ obtained after extrapolation of the resonant part back to time zero). A complete analysis of the data of Fig. 6.7 together with measurements at other polarization configurations has provided numbers for the components of the nonlinear susceptibility tensor $\chi_{\mathrm{NR}}^{(3)}$.

\section{c) Vibrational Transitions in Solids}

Time-resolved coherent Raman scattering supplies valuable dynamical information on vibrations (phonons) in the solid phase at low temperatures. Here the relaxation times are often long, e.g. $T_{2} \simeq 10^{-10} \mathrm{~s}$, and the study of the corresponding line shapes (width $\Delta v / c \sim 0.1 \mathrm{~cm}^{-1}$ ) via spontaneous Raman scattering becomes difficult. On the other hand, time-resolved coherent experiments are readily made in this time domain. Due to the well-defined dispersion relations $\omega(k)$ of the phonons in crystalline solids, the time-resolved experiments require special care to properly adjust the polarization and propagation conditions to the symmetry of the investigated modes (see e.g. Giordmaine and Kaiser [6.1] and Velsko et al. [6.66]). 
The relaxation time $T_{2}$ of vibrational modes in crystals has been treated theoretically in a number of publications [6.9,56,67-79]. It could be shown that the pure dephasing times increase with decreasing temperature proportional to $\left(T_{\mathrm{D}} / T\right)^{7}$ for $T \ll T_{\mathrm{D}}$, where $T_{\mathrm{D}}$ is the Debye temperature [6.67]. On the other hand, the energy relaxation time $T_{1}$ approaches a finite value at low temperatures and therefore determines the total phase relaxation $1 / T_{2}=1 /\left(2 T_{1}\right)$ for $T \rightarrow 0$.

The equivalence of the coherent relaxation time $\tau$ to the energy relaxation times $T_{1}$ at low temperatures has been shown experimentally for molecular crystals, e.g. by echo experiments for pentacene in naphthalene and by measuring time-resolved spontaneous anti-Stokes Raman scattering for non-thermal LOphonons in GaAs at $77 \mathrm{~K}[6.9,80,81]$.

Time-domain coherent Raman investigations exist for a number of crystals. In an early investigation the TO lattice mode of diamond was found to have a relaxation time $T_{2} / 2$ of $3.4 \mathrm{ps}$ and $2.9 \mathrm{ps}$ at $77 \mathrm{~K}$ and $295 \mathrm{~K}$, respectively [6.82]. Another example is given in Fig. 6.7, where $\mathrm{GaP}$ is investigated. The slow exponential decay of the coherent signal allows one to deduce the lifetime of the LO-phonon mode of $\mathrm{GaP}$ at $403 \mathrm{~cm}^{-1} \mathrm{~A}$ value of $T_{2} / 2=6.7 \pm 0.3 \mathrm{ps}$ was reported [6.33]. In a recent paper, Bron et al. [6.83] studied the temperature dependence of $T_{2}$ of the LO-phonons of GaP and $\mathrm{ZnSe}$ by time-resolved coherent Raman scattering. The authors interpret their data as follows: At low temperatures the LO-phonons with wave vector $|k| \simeq 0$ decay into two acoustic phonons with half the energy and with equal wave vectors $q_{i}$ of opposite sign, $q_{1}=-q_{2}$. Impurity scattering was not relevant in the presence of nitrogen impurities of the order of $10^{16} \mathrm{~cm}^{-3}$ in the GaP crystal. For elevated temperatures $T>150 \mathrm{~K}$, higher-order phonon-phonon interactions begin to play a role.

The internal $A_{1}$ mode of $\alpha$-quartz at $v / c=465 \mathrm{~cm}^{-1}$ was investigated over a wide temperature range by Gale and Laubereau [6.84]. The experiments yielded a pronounced temperature dependence of the relaxation times, e.g. time constants of $T_{2} / 2=0.8$ and $3.4 \mathrm{ps}$ were found at 295 and $23 \mathrm{~K}$, respectively. The phonon relaxation in $\alpha$-quartz was discussed in terms of a three-phonon process, where the population of the excited $465 \mathrm{~cm}^{-1}$ phonon decays, generating an acoustic phonon at $v_{1}=51 \mathrm{~cm}^{-1}$ and an optical phonon at $v_{2}=414 \mathrm{~cm}^{-1}$ The temperature dependence of the decay time $T_{2}$ was successfully described by the relation $2 / T_{2}=\gamma\left[1+n_{1}(T)+n_{2}(T)\right]$, where $n_{1}$ and $n_{2}$ are the thermal occupation numbers of the created phonons, $\gamma$ is the low-temperature relaxation rate and $T$ the temperature.

Besides the examples given above numerous publications have focused on other inorganic crystals. They have treated, for example, the dephasing of onephonon states in calcite [6.30,85,86], of polaritons in ammonium chloride [6.87], or of two-phonon states [6.88-90].

A number of papers have addressed vibrational modes in organic molecular crystals [6.91-102]. In these materials the transition frequencies are often similar to those in molecular liquids. When several molecules are in the elementary cell, a splitting of the transition lines may occur due to the different local symmetry 


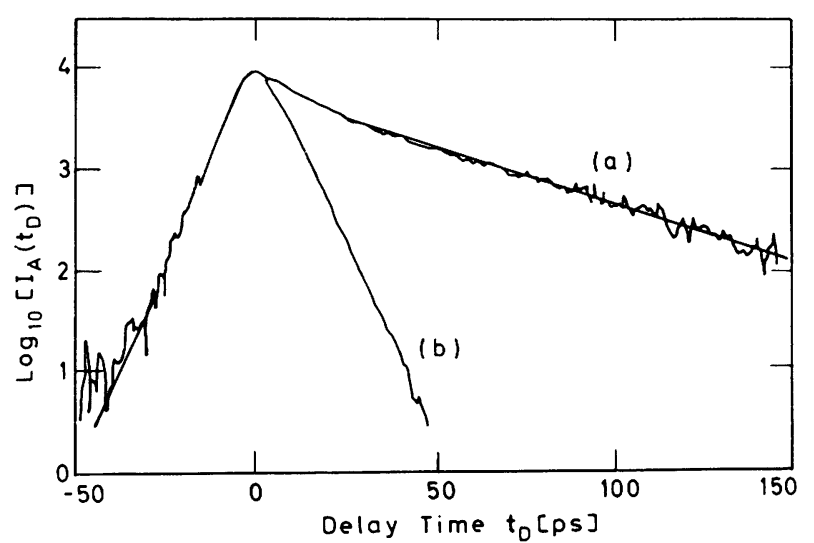

Fig. 6.8. Time-resolved CARS signal decay for the $v_{1}$ mode, $A_{\mathrm{g}}$ factor group of crystallized benzene of natural isotope composition at $1.6 \mathrm{~K}$. (a) Measured signal and best-fit curve. From the exponential decay a time constant of $T_{2} / 2=40 \pm 2 \mathrm{ps}$ is deduced. (b) Instrumental response function [6.97]

of the molecules (factor group splitting). Extensive investigations of the relaxation processes in crystalline benzene were carried out by Hochstrasser's group [6.96-101].

An example is given in Fig. 6.8, curve $a$, where the coherent anti-Stokes signal at $1.6 \mathrm{~K}$ is plotted as a function of the time delay $t_{\mathrm{D}}$ for the $A_{1 \mathrm{~g}}$ factor group of the $v_{1}$ mode $\left(v / c=991 \mathrm{~cm}^{-1}\right)$ of benzene with natural carbon isotopic composition (curve $b$ gives the instrumental response) [6.97]. The signal decays exponentially with a time constant of $\tau=T_{2} / 2=40 \pm 2$ ps at later delay times. Extrapolating the exponential slope back to time zero one finds a slight contribution from the nonresonant susceptibility $\chi_{\mathrm{NR}}^{(3)}$. A number of additional experiments were carried out with benzene at low temperatures in order to deduce the relevant relaxation mechanism. Measurements of different factor groups indicated faster decay times of $\tau=35.6$ ps for the $B_{2 \mathrm{~g}}$ component [6.99]. Mixed crystals containing $\mathrm{C}_{6} \mathrm{D}_{6}$ molecules gave increased decay rates [6.97]. Important for the understanding of the relaxation mechanism is the finding that the decay rates depend on the isotopic composition of carbon in the benzene molecule. It should be noted that natural benzene contains $1.1 \%$ of ${ }^{13} \mathrm{C}$ carbon. Experiments have been performed with neat ${ }^{12} \mathrm{C}_{6} \mathrm{H}_{6}$ benzene crystals giving considerably longer decay times of $T_{2} / 2=61.7 \mathrm{ps}\left(A_{1 \mathrm{~g}}\right)$ and $T_{2} / 2=56 \mathrm{ps}\left(B_{2 \mathrm{~g}}\right)$. Strong isotope effects have also been found for a number of other vibrational modes of benzene crystals [6.99].

The low-temperature relaxation rates of the isotopically pure benzene crystals show mode specific energy relaxation. The ${ }^{13} \mathrm{C}$ "impurities" in the molecules cause increased relaxation rates due to impurity phonon scattering or near resonant $\left(\Delta E \sim 9 \mathrm{~cm}^{-1}\right)$ energy trapping. The experiments with benzene crystals demonstrate that the relaxation rates measured via time-resolved coherent Raman scattering give a lower value for the energy relaxation time $T_{1}$ in the isotopically mixed samples, while they give $T_{1}$ in the pure crystals. These observations provide convincing evidence that the isotopic composition of the molecules has to be taken into account when interpreting the phonon relaxation. 

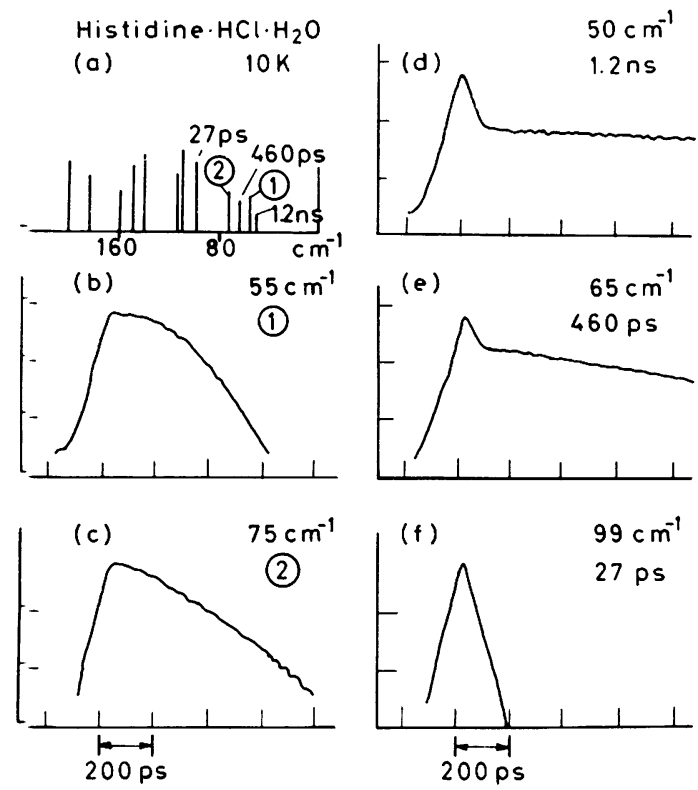

Fig. 6.9a-f. Time-resolved coherent Raman data for $1-(t)$-histidine. $\mathrm{HCl} \cdot \mathrm{H}_{2} \mathrm{O}$ crystals at $10 \mathrm{~K}$ (semi$\log$ plots). (a) Positions of the lowfrequency modes. (b)--(f) Timeresolved data for the five lowfrequency modes shown in (a). Exponential decays are found for the totally symmetric modes at 99 $\mathrm{cm}^{-1}, 65 \mathrm{~cm}^{-1}$, and $50 \mathrm{~cm}^{-1}$, whereas the two modes at $55 \mathrm{~cm}^{-1}$ (b) and $75 \mathrm{~cm}^{-1}$ (c) suggest inhomogeneous line-broadening [6.102]

Of special interest are time-resolved coherent Raman investigations in large Inolecules which are of biological relevance. Extensive investigations of lowfrequency vibrational modes (librons) in crystals of aminoacids and peptides were reported by Dlott [6.79]. An example is presented in Fig. 6.9, where crystals of IL-histidine hydrochloride monohydrate were investigated at $10 \mathrm{~K}$ [6.102]. The frequency positions, the intensities, and the observed lifetimes are summarized schematically in Fig. 6.9a. A common feature of the relaxation properties of the vibrations in the aminoacid crystals is the following: Modes with frequencies higher than $150 \mathrm{~cm}^{-1}$ show lifetimes $\tau=T_{1}$ shorter than $10 \mathrm{ps}$. For the symmetric tnodes the lifetimes increase with decreasing frequency $\omega_{0}$. The relaxation data for the totally symmetric 99,65 , and $50 \mathrm{~cm}^{-1}$ lattice modes are depicted in Fig. (5.9d-f, where the lifetimes extend up to $\tau=1.2 \mathrm{~ns}$. From the frequency depenclence of $T_{1}$ the authors concluded that the decay times are determined by s.pontaneous decay of librons into two counterpropagating acoustic phonons or into one libron and one acoustic phonon. The non-totally symmetric local modes at 55 and $75 \mathrm{~cm}^{-1}$ show non-exponential (Gaussian) decay (see Fig. $6.9 \mathrm{~b}, \mathrm{c}$ ). The inhomogeneous broadening of these local modes is believed to be due to dipolar interactions or small variations in the crystal packing.

A short summary should be given here concerning the coherent relaxation times and the relaxation mechanisms found in molecular crystals:

i) Homogeneously broadened lines are frequently found. The inhomogeneity 'which one would expect due to the site-specific frequency shifts appears in most 
systems as factor group splitting. It is commonly accepted that imperfections o the crystals are less effective due to the delocalisation of the phonons (motiona narrowing); but they may strongly affect the relaxation processes.

ii) The frequency widths or time constants at elevated temperatures are determined by energy relaxation, dephasing or intraband relaxation, and im purity scattering. In a number of cases the temperature dependence of the time constant allows one to determine the relaxation mechanism.

iii) At very low temperatures the relaxation rates become constant. They ar determined by energy relaxation and impurity scattering. The decay times mea sured in coherent experiments give directly the energy relaxation time $T_{1}$. Cars has to be taken when impurities influence the coherent decay times; in this cas only a lower limit for $T_{1}$ may be estimated.

\section{d) The Collision Time}

We return now to molecular modes in liquids. The theoretical model of Sectior 6.1 treated the relaxation processes in terms of two time constants, $T_{1}$ and $T_{2}$ At very early times, $t_{\mathrm{D}} \ll T_{2}$, however, the time-dependent interaction durin\& the collisions of the molecules becomes important. As a consequence, the correla tion function $\langle\langle q(0) q(t)\rangle\rangle$ is no longer a single exponential, but has the form $[6.3,4,50,103]$ :

$$
\langle\langle q(0) q(t)\rangle\rangle \propto \exp \left\{-\frac{t}{T_{2}}-\frac{\tau_{\mathrm{c}}}{T_{2}}\left[\exp \left(-\frac{t}{\tau_{\mathrm{c}}}\right)-1\right]\right\} .
$$

Equation (6.15) was derived by Kubo for an exponential decay of the fre quency correlation function, $\langle\delta \omega(t) \delta \omega(0)\rangle=\exp \left(-t / \tau_{\mathrm{c}}\right)$. The collision time $\tau$. is the time for which a certain frequency shift $\delta \omega(t)=\omega(t)-\omega_{0}$ is maintained.

At later times (6.15) gives the exponential decay of the coherent amplitude with $\exp \left(-t / T_{2}\right)$, while at short times, $t \rightarrow 0,(6.15)$ implies a Gaussian time dependence $\exp \left[-t^{2} /\left(T_{2} \tau_{\mathrm{c}}\right)\right]$. For short collision times $\tau_{\mathrm{c}} \ll T_{2}$ the collisior process leads to small corrections of the coherent signal. Nevertheless, time resolved coherent scattering may be used to determine the collision time $\tau$, or can enable one to estimate an upper limit for $\tau_{\mathrm{c}}$. For an example we refer tc Figs. $6.4 \mathrm{~b}$ and 6.5 . The decay of the coherent signal was found to be exponentia; for times larger than $0.3 \mathrm{ps}$. Taking into account the accuracy of the experimenta: data and the nonresonant susceptibility $\chi_{\mathrm{NR}}^{(3)}$, one may estimate an upper limit for the collision time of both acetone modes and for the acetonitrile mode o: $\tau_{\mathrm{c}}<0.3 \mathrm{ps}$.

For sufficiently long collision times $\tau_{\mathrm{c}} \geqslant 0.5 \mathrm{ps}$, picosecond light pulses from a Nd:glass laser may be used for the direct determination of $\tau_{\mathrm{c}}$. Telle and Laubereau have performed coherent experiments with a carefully controlled time resolution of the experiment [6.104]. They simultaneously measured the temporal response of their system (by coherent scattering of a purely nonresonant suscept- 
ibility) and the coherent signal of the investigated transition (liquid $\mathrm{CH}_{2} \mathrm{BrCl}$ ). From the experimental curves and numerical calculations based on the (measured) temporal system response the authors estimated a collision time of

$$
t_{\mathrm{c}}=0.4\left(\begin{array}{c}
+0.4 \\
-0.1
\end{array}\right) \mathrm{ps}, \quad \text { i.e. } 0.3-0.8 \mathrm{ps} .
$$

In a recent paper Chesnoy investigated the kinetics of vibrational dephasing of nitrogen under supercritical conditions [6.41]. He demonstrated that, after some initial delay, the coherent signal decays exponentially with time. Comparing the time-resolved coherent Raman data with spontaneous Raman spectra enabled a collision time of $\tau_{\mathrm{c}} \simeq 10 \mathrm{ps}$ to be deduced; this is of the same order as the measured dephasing time of $T_{2} \simeq 20 \mathrm{ps}$. The long collision time $\tau_{\mathrm{c}}$ indicates the importance of density fluctuations as a source of frequency modulation close to the critical point.

\subsubsection{Time-Resolved Coherent Raman Scattering of a Distribution of Resonance Frequencies}

\section{a) Time-Domain Experiments}

In the previous sections we discussed time-resolved coherent investigations, where dynamic processes such as dephasing and energy relaxation were of major relevance. Here we address new spectroscopic studies which allow accurate measurement of frequency differences between vibrational modes and the resolution of transitions within congested spectral regions.

The following time-resolved coherent experiments are described by (6.1-13) discussed above. For each vibrational mode one introduces individual coherent amplitudes $Q_{j}$ and vibrational resonance frequencies $\omega_{j}$. The coherently scattered field is a sum of field components scattered from the individual molecular modes $Q_{j}[6.7,37,105]$.

$$
E_{\mathrm{A}}\left(t, t_{\mathrm{D}}\right)=\sum_{j} E_{\mathrm{A} j}\left(t, t_{\mathrm{D}}\right) \propto \sum_{j} Q_{j}(t) E_{\mathrm{L} 2}\left(t-t_{\mathrm{D}}\right) \exp \left(-\mathrm{i} \omega_{j} t\right) .
$$

The observed coherent signal has the form $S\left(t_{\mathrm{D}}\right) \propto \int d t\left|E_{\mathrm{AS}}\left(t, t_{\mathrm{D}}\right)\right|^{2}$. It can be shown that in the limit of short excitation and probing pulses the signal $S\left(t_{\mathrm{D}}\right)$ at $t_{\mathrm{D}}>0$ is proportional to the absolute square of the Fourier transform of the spontaneous Raman spectrum $R(\omega)$, i.e. $S\left(t_{\mathrm{D}}\right) \propto\left|\int d \omega \exp \left(\mathrm{i} \omega t_{\mathrm{D}}\right) R(\omega)\right|^{2}[6.3,105]$. In the time domain - according to (6.16) - simultaneously excited modes lead to a beating of the coherent signal at the frequency differences $\delta \omega_{i j}$ between the various transition frequencies $\omega_{i}$ and $\omega_{j}$, with $\delta \omega_{i j}=\omega_{i}-\omega_{j}$. These "quantum" beats of the coherent signal were found for the first time by Laubereau et al. [6.36] in a number of tetrahalides. Later, similar beating phenomena were reported in several publications $[6.37,44,94,98,102,106-108]$. 


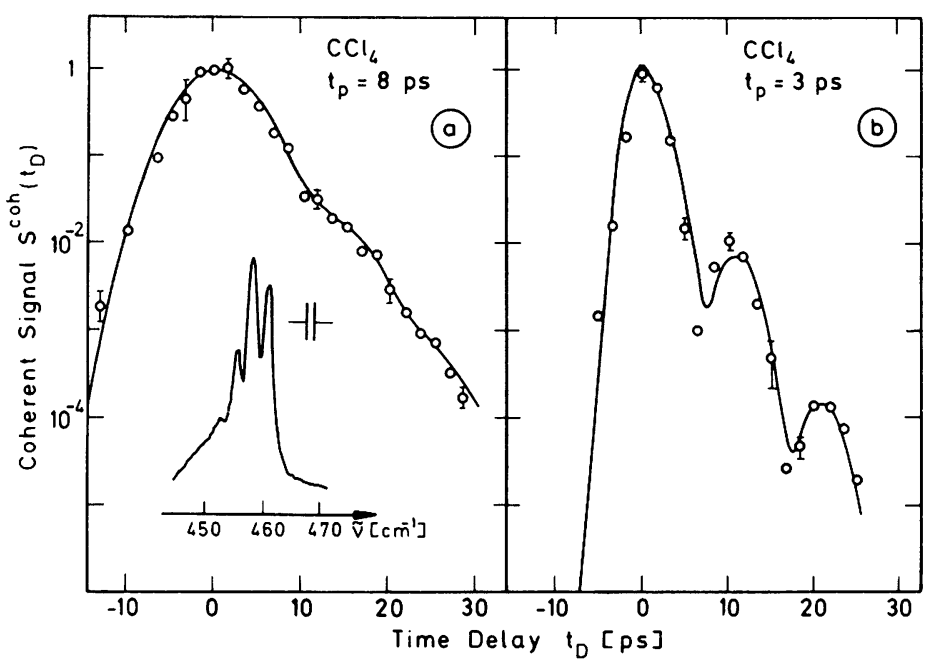

Fig. 6.10a, b. Coherent Stokes signal from the $v_{1}$ mode of liquid $\mathrm{CCl}_{4}$. The isotope composition of $\mathrm{CCl}_{4}$ leads to a splitting of the $v_{1}$ band in the spontaneous Raman spectrum - see insert in (a). The different resonance frequencies lead to the beating phenomenon which is clearly resolved in (b) where exciting and probing pulses of $3 \mathrm{ps}$ duration were used. For longer $\left(t_{\mathrm{p}}=8 \mathrm{ps}\right)$ pulses the beating structure is smeared out. Nevertheless, the dephasing time $T_{2}$ is readily determined in both experiments [6.57]

Figure 6.10 presents the results of a study of the $v_{1}$ mode of liquid carbontetrachloride $\left(v / c \simeq 459 \mathrm{~cm}^{-1}\right)$ where the influence of the pulse duration on the observed beating was investigated [6.37]. Due to the natural isotopic composition of the $\mathrm{CCl}_{4}$ molecules (the two chlorine isotopes ${ }^{37} \mathrm{Cl}$ and ${ }^{35} \mathrm{Cl}$ have abundances of $24 \%$ and $76 \%$, respectively), the transition is split in five equidistant components (distance $\Delta v / c=3 \mathrm{~cm}^{-1}$ ). Four of the more abundant components are readily resolved in the spontaneous Raman spectrum as shown in the insert of Fig. 6.10a. In the time-resolved coherent experiment one expects a beating phenomenon with maxima separated by $\Delta t=1 / \Delta v \simeq 11 \mathrm{ps}$. This beating is indeed observed in Fig. 6.10b, where short excitation and probing pulses with $t_{\mathrm{p}}=3 \mathrm{ps}$ were used. In addition, one obtains information on the dephasing time $T_{2}$. The solid curve was calculated using the measured pulse parameters $\left(t_{\mathrm{p}}=\right.$ $3 \mathrm{ps}$, Gaussian shape), a difference of the neighboring frequency components of $3.0 \mathrm{~cm}^{-1}$, a dephasing time $T_{2}=6 \mathrm{ps}$ (assumed to be the same for all components) and the nonresonant susceptibility $\chi_{\mathrm{NR}}^{(3)}$. Figure 6.10a shows the signal curve for longer excitation and probing pulses $\left(t_{\mathrm{p}} \simeq 8 \mathrm{ps}\right)$. Here the beating phenomenon is smeared out and the decay, with the time constant $T_{2}=6 \mathrm{ps}$, appears quite clearly. The longer pulses influence the signal curve in two ways: (i) During the longer excitation process the most intense transition of the Raman spectrum is preferentially excited, and (ii) the long duration of the probing pulse gives a levelling of the modulation. The two experiments of Fig. 6.10 show convincingly 


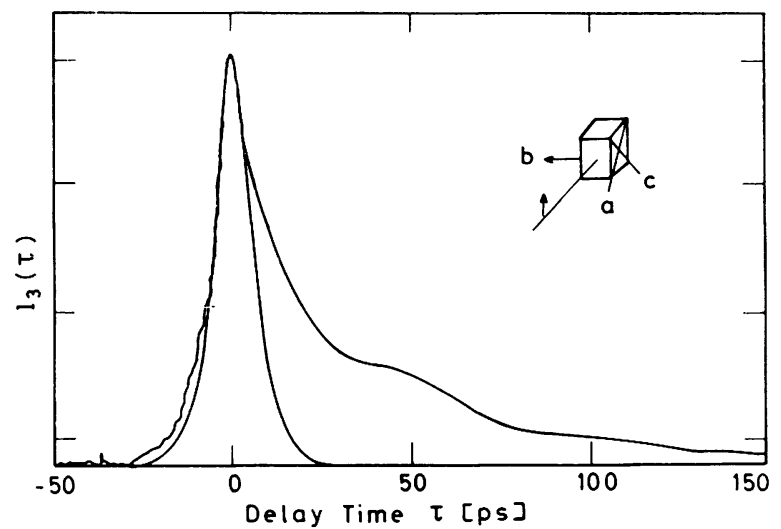

Fig. 6.11. Time-resolved coherent Raman data for a special phasematching condition, where the $A_{\mathrm{g}}$ and the $B_{2 \mathrm{~g}}$ factor group components of the $991 \mathrm{~cm}^{-1}$ vibron of crystallized benzene are probed simultaneously. The modulation of the signal is due to the frequency shift of $0.64 \mathrm{~cm}^{-1}$ between the two interfering lines. Also shown is the experimental response function [6.98]

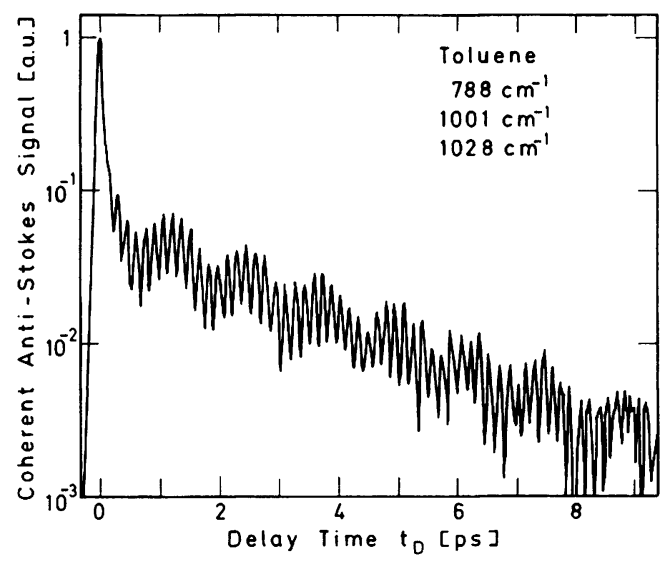

Fig. 6.12. Terahertz beats observed in timeresolved coherent Raman scattering from liquid toluene using femtosecond light pulses. The interference of the three simultaneously excited toluene modes at 788 , 1001 , and $1028 \mathrm{~cm}^{-1}$ generates a rich structure with beat frequencies up to $7.2 \mathrm{THz}$ [6.106]

that time-resolved coherent experiments are well suited to investigate frequency differences and decay times in spectral regions consisting of discrete frequency components.

Beating between transitions of different factor groups was studied by Velsko et al. [6.98] in crystallized benzene at low temperature (1.6 K). Some results are shown in Fig. 6.11. The geometry of the excitation and probing light field was selected in such a way that coherent signals were obtained from the $A_{\mathrm{g}}$ and $B_{2 \mathrm{~g}}$ states in the factor groups of the $991 \mathrm{~cm}^{-1}$ transition of benzene. From the beating of the signal (i.e. the time between the peaks) and from its magnitude the authors determined the frequency difference to be $\Delta v=19 \mathrm{GHz}$, the relative amplitudes to be $20: 1$, and the decay times of the two modes to be 40 and 34 ps, respectively.

While the experiment on crystallized benzene gave beating frequencies in the $20 \mathrm{GHz}$ range, the advent of femtosecond light pulses allows one to measure beating frequencies far into the terahertz regime. Beat frequencies of more than $10 \mathrm{THz}$ have been reported [6.44]. Figure 6.12 shows very recent data from a coherent Raman experiment on liquid toluene [6.106]. In the wide frequency 
range of $700-1100 \mathrm{~cm}^{-1}$ three vibrational modes are excited simultaneously by the (broadband) femtosecond light pulses. The scattered coherent anti-Stokes signal exhibits an amazing beating structure with beat frequencies of up to $7.2 \mathrm{THz}$. The frequency components of the signal curve are evaluated by Fourier transformation or other more specialized numerical methods in order to obtain the spectral information. This procedure called "Fourier transform Raman spectroscopy" was first demonstrated by Graener and Laubereau [6.107]. The authors studied coherent beating in gaseous methane-argon mixtures on a much longer time scale of many picoseconds to nanoseconds.

It should be noted here that the case of continuously distributed resonance frequencies (e.g. an inhomogeneously broadened transition) leads to a more difficult situation. There is no recurrence of the signal but only destructive interference with a rapid signal decay. An early attempt to measure $T_{2}$ in inhomogeneously broadened frequency distributions by using a "selectivity in $k$-space" had to be reconsidered [6.36,109-114]. Zinth et al. showed experimentally that with the existing dispersion of the samples, a selectivity for a molecular subgroup is not possible [6.37]. In addition, general theoretical arguments prove that the information from a single-pulse coherent experiment and from a spontaneous Raman spectrum is equivalent $[6.103,115,116]$; i.e. $T_{2}$ cannot be obtained in a coherent Raman experiment, where the spontaneous Raman line is strongly inhomogeneously broadened.

Under certain conditions, however, time-resolved coherent Raman techniques may give information on the line-broadening mechanism. Two processes cause similar non-Lorentzian spontaneous Raman bands: (i) Long collision times $\tau_{\mathrm{c}} \simeq T_{2}$ and (ii) inhomogeneous broadening (cross-relaxation times much longer than $T_{2}$ ).

Gale et al. [6.42] pointed out that coherent experiments give new information on the broadening mechanism: (i) If $\tau_{\mathrm{c}}$ is relatively long, $\tau_{\mathrm{c}} \simeq T_{2}$, the coherent signal decays non-exponentially around time zero but turns to an exponential decay at later times. (ii) For a small inhomogeneous contribution an initial exponential decay with time constant $T_{2} / 2$ (close to time zero) is followed by an increasingly rapid decay at later times [6.105]. If the inhomogeneous width adds $10 \%$ to the homogeneous linewidth, the slope of the signal decay (on a semilog plot) gives $T_{2} / 2$ around time zero. This slope increases with time. For example, four orders of magnitude below the signal peak, the slope is larger than at time zero by more than $50 \%$. While the influence of weak inhomogeneous broadening [case (ii)] and of long collision times $\tau_{\mathrm{c}}$ [case (i)] yield very similar spontaneous Raman spectra, the time-resolved coherent data are different and may be used to decide which line-broadening mechanism exists.

\section{b) Transient Frequency-Domain Experiments}

In the previous section we discussed investigations where the spectral information on the molecular system was deduced from time-resolved coherent data 
followed by a numerical analysis. Now we present a technique which avoids the Fourier transformation by directly measuring the spectrum of the coherent signal generated by properly shaped probing pulses $[6.64,117-121]$.

The basic idea of the technique is readily seen by considering the spectrum of the scattered coherent anti-Stokes signal of a single vibrational transition

$$
\begin{aligned}
& I_{\mathrm{A}}(\omega) \propto\left|\int d t \exp (\mathrm{i} \omega t) E_{\mathrm{A}}(t)\right|^{2} \\
& I_{\mathrm{A}}(\omega) \propto\left|\int d t \exp (\mathrm{i} \omega t) E_{\mathrm{L} 2}(t) \exp \left(-\frac{t}{T_{2}}\right)\right|^{2} \\
& I_{\mathrm{A}}(\omega) \propto\left|\int d t \exp (\mathrm{i} \omega t) \exp \left[-\left(\frac{t}{t_{0}}\right)^{2}-\frac{t}{T_{2}}\right]\right|^{2} \\
& I_{\mathrm{A}}(\omega) \propto\left|\int d t^{\prime} \exp \left(\mathrm{i} \omega t^{\prime}\right) \exp \left[-\left(\frac{t^{\prime}}{t_{0}}\right)^{2}\right]\right|^{2} .
\end{aligned}
$$

In these equations it is assumed that the excitation and the initial effects of the collision process are terminated. Equation (6.17a) represents the Fourier transform of the anti-Stokes signal. The freely relaxing coherent amplitude $Q=Q_{0} \exp \left(-t / T_{2}\right)$ is monitored by a Gaussian shaped laser field $E_{\mathrm{L} 2}(t) \propto$ $\exp \left(-t^{2} / t_{0}^{2}\right)$ [see $\left.(6.17 \mathrm{~b}, \mathrm{c})\right]$. By introducing a new time $t^{\prime}$, one eliminates the dephasing time. According to $(6.17 \mathrm{~d})$, the anti-Stokes spectrum has the same frequency shape, i.e. the same frequency bandwidth, as the probing laser pulse $E_{\mathrm{L} 2}$. With the phase factors included in (6.17) one finds the anti-Stokes band centered at the anti-Stokes frequency $\omega_{\mathrm{AS}}=\omega_{\mathrm{L} 2}+\omega_{0}$. When sufficiently long $\left(t_{\mathrm{p}}>1.5 T_{2}\right)$ probing pulses of Gaussian shape are used, the spectral width $\Delta v$ of the time-resolved coherent anti-Stokes band, $\Delta v=0.44 / t_{\mathrm{p}}$, is narrower than the spontaneous Raman line $\Delta v=1 / \pi T_{2}$. In practical applications it is advantageous to use a short exciting force and a prolonged Gaussian shaped interrogation pulse. As an acronym the spectroscopic technique is known as SEPI spectroscopy. A more detailed description of the theory is given in the literature by $Z$ inth et al. [6.119] and Collins et al. [6.122].

An experimental example of the line-narrowing SEPI spectroscopy is given in Fig. 6.13. The $v_{1}$ mode of liquid $\mathrm{CCl}_{4}$ was investigated using a $\mathrm{Nd}$ : glass laser system supplying $20 \mathrm{ps}$ probing pulses of Gaussian shape. In Fig. 6.13a the spontaneous Raman spectrum of the $v_{1}$ mode is shown as measured with a high-resolution Raman spectrometer; the isotopic structure has been discussed above. The SEPI spectrum (Fig. 6.13b) was taken by using a high-resolution spectrograph in conjunction with an optical spectrum analyser. The entire coherently scattered spectrum is recorded immediately for each probe pulse. When the excitation force (i.e. $E_{\mathrm{L}} E_{\mathrm{s}}^{*}$ ) is centered at $460 \mathrm{~cm}^{-1}$ (see Fig. $6.13 \mathrm{~b}$ ), the two high-frequency components of the isotopically split Raman band appear very narrow and thus well resolved. The SEPI spectrum clearly separates the two 

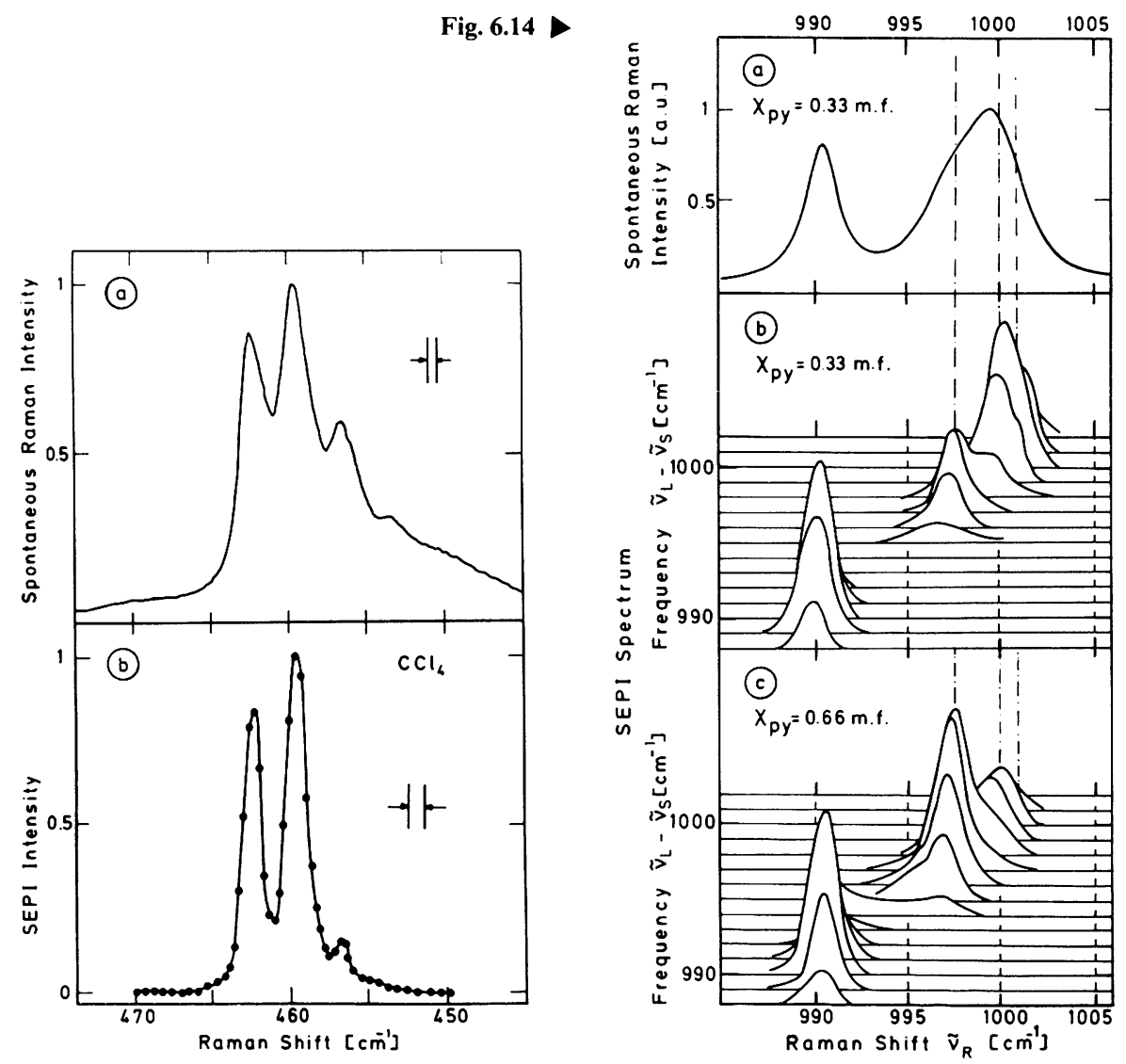

Fig. 6.13a, b. Line-narrowing coherent Raman spectroscopy using short excitation and prolonged coherent interrogation (SEPI) of the $v_{1}$ mode of liquid $\mathrm{CCl}_{4}$. Broad overlapping lines of the spontaneous Raman spectrum (a) are considerably narrowed by the coherent SEPI technique (b) [6.105]

Fig. 6.14a-c. Raman spectra of pyridine: methanol mixtures. (a) Polarized spontaneous Raman spectrum measured at a pyridine concentration of 0.33 molar fraction taken with a standard Raman spectrometer of spectral resolution of $0.5 \mathrm{~cm}^{-1}$. (b) and (c) Short excitation and prolonged interrogation (SEPI) spectra obtained for a set of excitation frequencies, $v_{\mathrm{L}}-v_{\mathrm{s}}$, at two pyridine concentrations, $\chi_{\mathrm{py}}=0.33$ molar fraction (b) and $\chi_{\mathrm{py}}=0.66$ molar fraction (c). The spectra taken at excitation frequencies $v_{\mathrm{L}}-v_{\mathrm{s}}<994 \mathrm{~cm}^{-1}$ are drawn with reduced amplitudes. The SEPI spectra resolve three Raman lines of different hydrogen-bonded aggregates hidden under the broad band of the spontaneous Raman spectrum (see dash-dotted lines) [6.120]

bands and allows a very accurate determination of the frequency difference of $\delta v / c=3.0 \pm 0.08 \mathrm{~cm}^{-1}$.

An interesting application of the line-narrowing SEPI spectroscopy is presented in Fig. 6.14 [6.120]. The molecular system pyridine: methanol was investigated, which exhibits a broad featureless spontaneous Raman band around 
$1000 \mathrm{~cm}^{-1}$ (see Fig. 6.14a). The position and amplitude of this band changes with varying methanol concentration. With the help of a picosecond dye laser system, the frequency of the excitation force was tuned over the frequency range of interest. SEPI spectra taken at fourteen excitation frequencies are shown in Fig. $6.14 \mathrm{~b}, \mathrm{c}$ for two different methanol concentrations. In both cases the band at $1000 \mathrm{~cm}^{-1}$ can be resolved in three components at $997.3,1000.0$, and $1001 \mathrm{~cm}^{-1}$. The positions of the three bands remain constant while the amplitudes depend on the methanol concentration. From these data new information on hydrogenbonded complexes between pyridine and methanol were obtained: there exist at least three distinct molecular complexes of pyridine and methanol with frequency positions remaining constant with methanol concentrations. The apparent frequency shift of the Raman band observed in spontaneous spectroscopy results from the changing abundance of the complexes as a function of the methanol concentration.

The following points concerning the line-narrowing transient Raman spectroscopy are of interest:

i) The SEPI method investigates the freely relaxing molecules.

ii) Influences of $\chi_{\mathrm{NR}}^{(3)}$ are eliminated as the probing process is performed at late delay times.

iii) We emphasize that the amplitudes of the SEPI bands depend on the Raman cross sections and also on the dephasing times $T_{2}$ of the individual modes. This fact provides increased spectral resolution in congested spectral regions when fast decaying bands disappear at late delay times.

iv) The SEPI experiments may be performed with coherent anti-Stokes or Stokes scattering. The two spectra give the same information.

\subsubsection{Resonant Pulse Propagation in the Infrared}

In the previous sections the discussion concentrated on Raman-type interactions. The absence of a dipole moment required a pair of light pulses for the excitation of the molecules and a separate probing pulse to study the time evolution of the material excitation. In this section we focus on infrared-active transitions where the material is excited by resonant ir light and where the oscillating molecules emit radiation. We discuss the case of a single small area excitation pulse propagating resonantly through the absorbing medium [6.7,123-125].

It has been shown in [6.7] that small area pulse propagation may be treated by a single equation containing the dephasing time $T_{2}$ and a modified Bessel function. The discussion may be further simplified for the case of optically thin samples, i.e. where the absorbance $a=\alpha l=$ (absorption coefficient $\times$ sample length) is small, $a \ll 1$. The transmitted electric field may then be written as follows [6.125]:

$$
E_{\mathrm{tr}}(t)=E_{0}(t)-\frac{\alpha l}{2 T_{2}} \int_{-\infty}^{t} d t^{\prime} E_{0}\left(t^{\prime}\right) \exp \left[\left(t^{\prime}-t\right)\left(\frac{1}{T_{2}}-\mathrm{i} \Delta\right)\right] .
$$


As previously, $\Delta$ stands for the difference between resonance and driving frequency. According to (6.18) the transmitted electric field consists of the incoming light field minus a second term which decays with the dephasing time $T_{2}$. Thus the dephasing time $T_{2}$ can be directly measured from the slope of the trailing part of the transmitted, i.e. coherently emitted, light field. In the general case of $\alpha l \gtrsim 1$ the trailing part of the signal decays more rapidly. For values $\alpha l=1$ a simple correction formula may be used in order to deduce $T_{2}$ from the measured decay [6.126].

An elegant experimental system to measure the small area pulse propagation in the infrared was developed by Hartmann and Laubereau [6.124]. The main difficulty which had to be overcome was the slow time resolution of the photodetectors. Existing infrared detectors are not able to measure the transmitted pulse with picosecond time resolution. The detection of the pulse intensity as a function of time is accomplished by gated ultrafast infrared up-conversion. This technique provides the convolution of the instantaneous infrared intensity with a short probing pulse. Tuning the time delay between the exciting infrared pulse and the probing gate pulse allowed the evolution of the coherently radiated infrared signal to be followed.

In a recent experiment, the coherent interaction of an infrared pulse with rotational transitions of $\mathrm{HCl}$ : Ar gas mixtures at medium pressure was studied [6.127]. $\mathrm{HCl}$ was used with natural isotope abundance of the ${ }^{35} \mathrm{Cl}$ and ${ }^{37} \mathrm{Cl}$ isotopes. Transitions of the $R(J)$ branch of $\mathrm{HCl}$ around $3000 \mathrm{~cm}^{-1}$ were investigated for different values of the rotational quantum number $J$ of the $J \rightarrow J+1$ rotation-vibrational transition. An example is presented in Fig. 6.15. The infrared

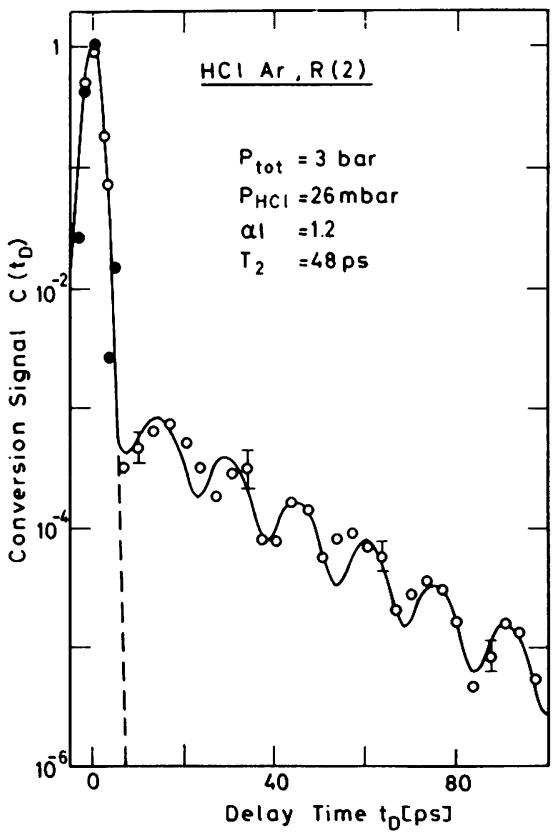

Fig. 6.15. Resonant propagation of an infrared pulse through a $\mathrm{HCl}: \mathrm{Ar}$ mixture. The conversion signal representing the transmitted and reemitted infrared intensity is plotted versus delay time $t_{\mathrm{D}}$. Note the beating in the reemitted part at $t_{\mathrm{D}}>10 \mathrm{ps}$ which is due to the interference from the emission from the $R(2)$ transition of $\mathrm{HCl}$ containing the two chlorine isotopes ${ }^{35} \mathrm{Cl}$ and ${ }^{37} \mathrm{Cl}$. From the decay of the signal a dephasing time $T_{2}=48 \pm 5 \mathrm{ps}$ was determined [6.127] 
pulse is tuned to the $R(2)$ transition of $\mathrm{H}^{35} \mathrm{Cl}$. The related $R(2)$ transition of $\mathrm{H}^{37} \mathrm{Cl}$ is shifted by $\simeq 2 \mathrm{~cm}^{-1}$ to smaller frequencies but also interacts with the incident pulse. [For the numerical analysis of the experimental data the second transition has to be incorporated in (6.18)]. The time integrated conversion signal $C\left(t_{\mathrm{D}}\right)$ is plotted in Fig. 6.15 on a logarithmic scale as a function of the delay time of the probing pulse. Around $t_{\mathrm{D}}=0$ the conversion signal of the emitted pulse (open circles, solid line) follows the input pulse (full circles, broken line). For longer delay times, $t_{\mathrm{D}}>10 \mathrm{ps}$, the emitted pulse decays more slowly. A beating phenomenon (beat time $15.3 \mathrm{ps}$ ) is found which is superimposed on an approximately exponential decay $(\tau=19.5 \mathrm{ps})$. The analysis of the data - taking into account the optical thickness of the sample, $\alpha l=1.2$ - yielded a frequency distance between the isotope components of $2.18 \mathrm{~cm}^{-1}$ and the dephasing time of $T_{2}=48 \pm 5$ ps. Measurements at different partial pressures of $\mathrm{HCl}$ and $\mathrm{Ar}$ gave the following results: $\mathrm{HCl}-\mathrm{HCl}$ collisions are much more efficient for the dephasing process than $\mathrm{HCl}-\mathrm{Ar}$ collisions; the relaxational data for the rotational-vibrational transitions of $\mathrm{HCl}$ agree well with the relaxation data for pure rotational transitions, suggesting that vibrational relaxation contributes little to the observed dephasing process. The authors proposed that total dephasing is due to pure rotational dephasing (quasi-elastic collisions) and the population decay of the rotational levels (inelastic collisions).

\subsection{Coherent Spectroscopy Using Multiple Excitation Processes}

In the preceding sections we have discussed various applications of coherent spectroscopy using a single excitation process. One major result was the determination of the dephasing time $T_{2}$ in a variety of systems. On the other hand, these techniques do not allow measurement of the dephasing time $T_{2}$ for wide inhomogeneously broadened lines and give no information on the energy relaxation time $T_{1}$ (except in solids at low temperature).

In the theoretical part (Sect. 6.1.3) it was shown that multiple excitation experiments provide additional information. These experiments, frequently called echo experiments, have their analogues in magnetic resonance. They have been demonstrated for optical transitions, initially on a nanosecond or even longer time scale [6.11-13]. More recently, picosecond and femtosecond measurements have been reported. Successful echo experiments require a pulse area $A$ of approximately one. For small pulse areas, the echo signal is drastically reduced, since the signal intensity is proportional to $A^{6}$. For this reason ultrafast echo experiments are only possible when the relaxation times $T_{1}$ and $T_{2}$ are sufficiently long and when the transition dipole moment $\mu$ is large. Since 1975 ultrafast echo experiments have been performed on electronic transitions in dye solutions [6.128-130], in low-temperature solids (e.g. excitons in semiconductors [6.131, $132]$ and molecular crystals $[6.9,133-139]$ on a picosecond time scale or, more 
recently, in dye solutions with femtosecond pulses [6.140-142]. A number of theoretical papers treat the different echo techniques [6.143-147].

In the literature different - and in some cases misleading - names have appeared for the same coherent experiment. We briefly discuss (i) the echo experiments and (ii) the induced grating experiments, two aspects of the same coherent investigation.

i) In a coherent experiment with multiple excitation pulses, the system is excited (at time zero) by a first pulse (wave vector $\boldsymbol{k}_{a}$ ). After an evolution time $t_{1}$ a second excitation pulse (wave vector $\boldsymbol{k}_{b}$ ) passes through the sample and interacts with the excited molecules. Rephasing of the excited molecule leads (in the case of inhomogeneous broadening) to the formation of a delayed radiation the echo. The echo is emitted at a time $t=t_{1}$ after the second pulse under the well-defined phase-matching condition with wave vector $\boldsymbol{k}_{\mathrm{e}}=2 \boldsymbol{k}_{b}-\boldsymbol{k}_{a}$.

ii) In the excitation process, the polarization produced by a first excitation pulse and part of the second pulse, form an excitation grating in the sample (the induced grating) with the wave vector $\boldsymbol{k}_{\mathrm{g}}=\boldsymbol{k}_{\boldsymbol{b}}-\boldsymbol{k}_{a}$. The trailing part of the second pulse or a third pulse (see Sect. 6.3.2) interacts with the grating, generating radiation at the wave vector $\boldsymbol{k}_{\mathrm{s}}=\boldsymbol{k}_{b}+\boldsymbol{k}_{\mathrm{g}}=2 \boldsymbol{k}_{b}-\boldsymbol{k}_{a}$. This radiation - which forms the echo - is emitted with a time delay of $t=t_{1}$ in the case of inhomogeneously broadened transitions.

\subsubsection{Ultrafast Two-Pulse Echo Spectroscopy}

In the theoretical part [Eq. (6.7) in Sect. 6.1.2] a formal solution for the evolution of a two-level system was derived for the limiting case of light pulses with duration $t_{p}$ much shorter than the system's time constants $T_{1}$ and $T_{2}$. In the case of a two-pulse echo, two pulses of area $A_{a}$ and $A_{b}$ are applied to the sample with a time separation of $t_{1}$. The resulting echo polarization is given by (6.9). The signal amplitude is proportional to $\sin A_{a} \sin ^{2}\left(A_{b} / 2\right)$ and decays with the dephasing time $T_{2}$. The emission direction is determined by the wave vector $k_{\mathrm{e}}=2 k_{b}-$ $k_{a}$. An important point emerges from the underlined part of (6.9): in the case of a wide inhomogeneously broadened transition the signal is emitted close to time $t=t_{1}$, i.e. the emission occurs delayed as a photon echo.

In a standard two-pulse echo experiment the echo energy, i.e. the time integral of the absolute square of the echo amplitude, is measured as a function of the separation $t_{1}$ between the two excitation pulses. In the case of a broad inhomogeneous line the signal is emitted only close to $t=t_{1}$ and its intensity decays with $\exp \left(-4 t_{1} / T_{2}\right)$. For homogeneously broadened transitions the signal is emitted starting at $t=0$. The decay (as a function of $t_{1}$ ) follows $\exp \left(-2 t_{1} / T_{2}\right)$. (For intermediate line broadening see [6.14]). In order to obtain the dephasing time independently of the nature of the line broadening, only the polarisation at the time $t_{\mathrm{e}}=t_{1}$ should be measured (this may be done by optical gating techniques). Under these conditions the signal depends on $t_{1}$ as $\exp \left(-4 t_{1} / T_{2}\right)$ and is independent of the line shape. 
The preceding discussion shows that the two-pulse echo technique allows determination of the dephasing time $T_{2}$ even for inhomogeneously broadened transitions, where experiments with single excitation pulses fail.

A number of two-pulse echo experiments have been performed on exciton bands of molecular crystals at low temperature. For instance, the relaxation of the 0-0 transition of pentacene in naphthalene (and in p-terphenyl) was studied as a function of temperature by Hesselink and Wiersma $[6.9,133]$. Here the energy relaxation times $T_{1}$ are much longer $(\simeq 20 \mathrm{~ns})$ than the dephasing times $T_{2}$ and the pure dephasing times $T_{2}^{*}$ could be readily determined from the relation $1 / T_{2}=1 / T_{2}^{*}+1 /\left(2 T_{1}\right) \simeq 1 / T_{2}^{*}$. The temperature dependence of $T_{2}^{*}$ was fitted with a relaxation theory involving low-energy $\left(v / c \simeq 16 \mathrm{~cm}^{-1}\right)$ pseudo-local phonons coupled to the pentacene transition. The experiment clearly demonstrated the importance of this relaxation channel for the dephasing of the excitonic transitions.

Recently, the two-pulse echo technique has been applied to two-dimensional excitons in GaAs-AlGaAs quantum wells by Schultheis et al. [6.132]. The authors used a synchronously pumped dye laser emitting pulses (with $12.6 \mathrm{ps}$ autocorrelation width) tunable in the range of the exciton absorption band between $\lambda=820 \mathrm{~nm}$ and $\lambda=750 \mathrm{~nm}$. A pair of pulses at the temporal separation $t_{1}$ with wave vectors $\boldsymbol{k}_{a}$ and $\boldsymbol{k}_{b}$ was focussed into a sample consisting of 78 alternating layers of GaAs and $\mathrm{AlGaAs}$ (thickness $102 \AA$ and $200 \AA$, respectively), grown by molecular beam epitaxy. The sample was kept at $2 \mathrm{~K}$. The transmission spectrum of the inhomogeneously broadened exciton band is shown in the insert of Fig. 6.16 (broken curve). The echo signal was detected in the phasematching direction $2 \boldsymbol{k}_{b}-\boldsymbol{k}_{a}$ with the help of a lock-in system. A series of data is shown in Fig. 6.16. The echo signals are plotted as a function of delay time $t_{1}$ for three different energies of the excitation photons. Curve $a$ investigates the exciton at the low-energy side of the absorption band, curve $b$ was measured at somewhat higher energies, and curve $c$ was taken close to the peak of the exciton absorption.

The relaxation times of the sample do not fulfill the limit of $T_{2} \ll t_{\mathrm{p}}$ as assumed in the theoretical discussion of (6.19). A more detailed analysis was required in order to derive the relaxation time $T_{2}$ from the experimental curve. Together with $T_{1}$-data from hole-burning experiments, the analysis yielded interesting numbers. Close to the peak of the exciton band (curve $c$ ) the relaxation times are fast; $T_{2} \simeq 4 \mathrm{ps}$ and $T_{1}=8 \mathrm{ps}$. Consequently, the diffracted signal intensity is small and the signal decay is very rapid. At the low-energy side of the exciton band the energy relaxation time $T_{1}$ is much longer (100 ps), while the dephasing increases to $22 \mathrm{ps}$. There is no change of the time constants $T_{1}$ and $T_{2}$ at the frequency positions $a$ and $b$ in the low-energy tail of the exciton band. The results are interpreted as follows: Near the peak of the exciton band, there are several contributions to the coherent decay: scattering with acoustic phonons, impurities and well thicknesses (spectral diffusion). Below the absorption peak, excitation is more localized and spectral diffusion contributes less to the dephasing pro- 


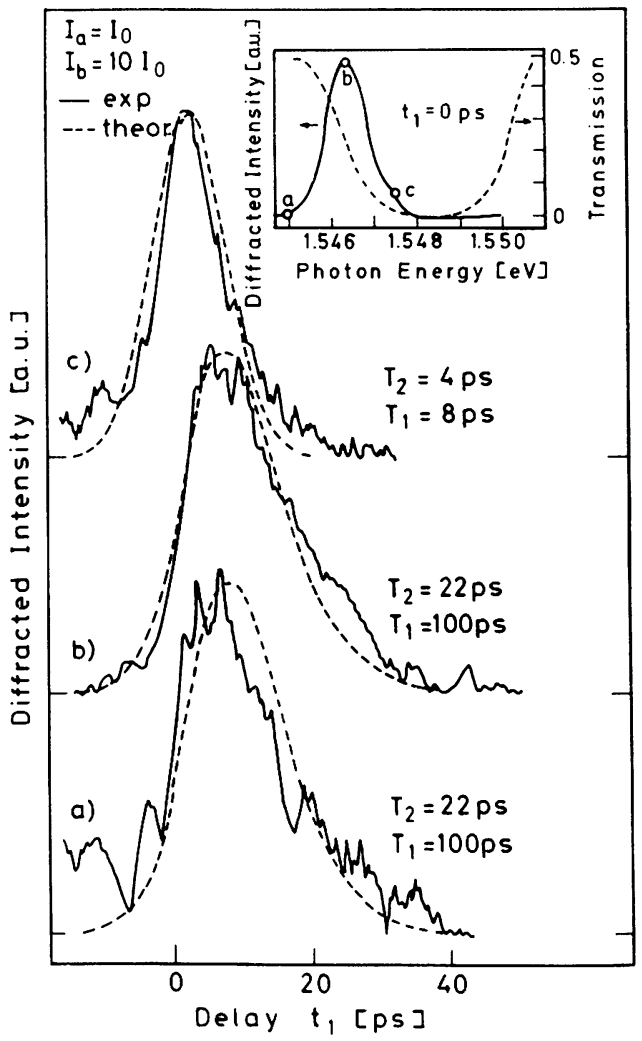

Fig. 6.16. Two-pulse photon echo experiments from two-dimensional excitons in GaAs-AlGaAs quantum wells. Experimental $(-)$ and theoretical (---) scattered intensity as a function of the delay time $t_{1}$ for three different photon energies. The data show different relaxation times $T_{2}$ depending on the spectral position of the excitation pulse within the heavy-hole exciton transition. The insert gives the scattered intensity at zero delay (solid curve) together with the transmission spectrum (dashed curve) [6.132]

cess. Very recent investigations of high-quality single quantum wells show a homogeneously broadened exciton band. Under these conditions acoustic phonon scattering is found to be the dominant broadening mechanism of the $2 D$ excitons [6.148].

\subsubsection{Three-Pulse Echos}

Experiments with three incident pulses constitute an important extension of the preceding coherent techniques. A schematic of this method was shown in Fig. 6.1c. Adding a third excitation pulse - which enters the sample at a time $t_{2}$ after the second pulse - gives new possibilities for studying molecular systems. For a two-level system, the three-pulse technique allows one to determine $T_{1}$ in a coherent experiment by varying the delay time $t_{2}$. When other molecular levels are involved in the relaxation process, a third pulse at a wavelength differing from that of the first two excitation pulses gives information on the path of the energy decay.

The three-pulse echo is calculated for a two-level system by solving (6.7) for the appropriate pulse sequence. The solution for the polarization $P(t)$ contains 
a number of different terms, which describe (i) the single-pulse excitation due to pulses $a, b$, and $c$, radiating in the directions $\boldsymbol{k}_{a}, \boldsymbol{k}_{b}$, and $\boldsymbol{k}_{c}$, respectively, (ii) two-pulse echos originating from pairs of excitation pulses radiating in the direction $2 \boldsymbol{k}_{\boldsymbol{i}}-\boldsymbol{k}_{j}$ for the various pulses $i, j=a, b, c$, (iii) higher-order terms of the order $A^{5}$, and finally (iv) the three-pulse echo:

$$
\begin{gathered}
P_{3 \mathrm{PE}}(t) \propto \int d \omega f(\omega) \exp \left[-\mathrm{i} \omega\left(t-t_{1}\right)-\left(t_{1}+t\right) / T_{2}\right] G\left(T_{1}, t_{2}\right) \\
\times \sin A_{a} \sin A_{b} \sin A_{c} \exp \left[-\mathrm{i}\left(\boldsymbol{k}_{b}+\boldsymbol{k}_{c}-\boldsymbol{k}_{a}\right) r\right]
\end{gathered}
$$

where $G\left(T_{1}, t_{2}\right)$ depends on the level scheme involved. For a closed system consisting of only two states $|a\rangle$ and $|b\rangle, G\left(T_{1}, t_{2}\right)$ has the form $G^{\mathrm{c}}\left(T_{1}, t_{2}\right)=$ $\exp \left(-t_{2} / T_{1}\right)$. For an open system, where the upper level decays to a long-lived $\left(\tau \gg t_{1}, t_{2}, t\right)$ state, which is not probed, $G\left(T_{1}, t_{2}\right)$ is $G^{0}\left(T_{1}, t_{2}\right)=1+\exp \left(-t_{2} / T_{1}\right)$. Equation (6.19) suggests a variety of possibilities for applying the three-pulse echo technique.

1) Variation of the excitation time $t_{1}$ allows the determination of the dephasing time $T_{2}$ in the case of inhomogeneously and homogeneously broadened transitions (in the same way as in the two-pulse echo experiment).

2) Variation of the time $t_{2}$ gives access to the population decay of the upper state. The experimentally observed kinetics depend on the details of the molecular system. If a closed two-level system is investigated, the coherent signal intensity decays with $\exp \left(-2 t_{2} / T_{1}\right)$. A more complex time dependence is observed for an open system where the decay of the signal is proportional to $\left[1+\exp \left(-t_{2} / T_{1}\right)\right]^{2}$; i.e. one observes a biexponential decay with decay times $T_{1} / 2$ and $T_{1}$, and the signal shows a constant background. The repopulation of the lower level $|b\rangle$ may also be deduced from the later kinetics.

3) The constant background, which is found due to the long-lived intermediate state in open systems allows accumulation of the echo by a sequence of excitation pulse pairs with a repetition rate faster than the spectral crosscorrelation time or the repopulation of the ground state. The accumulation of the grating may become so efficient that even weak cw mode-locked lasers can be used to induce a grating and to stimulate an echo.

4) Other aspects of the three-pulse echo technique may be inferred from the grating picture of the three-pulse echo experiment. The first two exciting pulses induce a population grating for the two levels coupled by the two pulses. The populations of the ground and excited state are changed. Energy transfer of either of the two grating states can be monitored. Using the third pulse at a new wavelength, it becomes possible to selectively monitor the relaxation processes. An example of such a two-color echo will be given below.

The laser systems for three-pulse or induced-grating experiments are $\mathrm{cw}$ mode-locked dye lasers with a repetition rate of $\sim 100 \mathrm{MHz}$ and a mean power of $30 \mathrm{~mW}$. In some cases, amplified pulses from $\mathrm{cw}$ mode-locked dye lasers at 
repetition rates of $10-100 \mathrm{~Hz}$ were applied. For high repetition rate lasers it is advantageous to use perpendicular polarizations for the excitation and probing process in order to remove artifacts due to temperature changes.

\section{a) Measurements of Energy Relaxation Times}

A number of interesting grating experiments were performed by Ippen's group using femtosecond light pulses [6.140-142]. In Fig. 6.17 the three-pulse echo experiment was done with amplified $75 \mathrm{ps}$ pulses $(\lambda=620 \mathrm{~nm})$ from a colliding pulse mode-locked ring dye laser (repetition rate of the amplified pulses $10 \mathrm{~Hz}$ ) [6.147]. The first two excitation pulses with wave vectors $k_{a}$ and $k_{b}$ entered the sample simultaneously, i.e. $t_{1}=0$. The scattered energy induced by a third pulse is plotted in Fig. 6.17 as a function of the delay time $t_{2}$. Two different samples, the dyes cresyl violet and oxazine 720 , were investigated and the results are shown in Fig. 6.17a and b, respectively. Both dyes were embedded in a thin film of a polymethylmethacrylate (PMMA) polymer and held at $15 \mathrm{~K}$. The excitation laser pulses populate vibronic levels in $S_{1}$, the first excited electronic state of the dyes. The first decay of the signal occurs on a time scale of several $100 \mathrm{fs}$. It results from an energy redistribution within the electronic state of the dye molecules. Similar rapid relaxation processes were found for dye molecules in solutions at room temperature [6.149]. At later times $t_{2}$, longer than $2 \mathrm{ps}$, a constant level of the signal is approached which decays within nanoseconds with the $S_{1}$ lifetime. The different amplitudes of the slower component found for the two dyes suggest complicated relaxation processes. They cannot be explained by simple closed or open two-level systems.
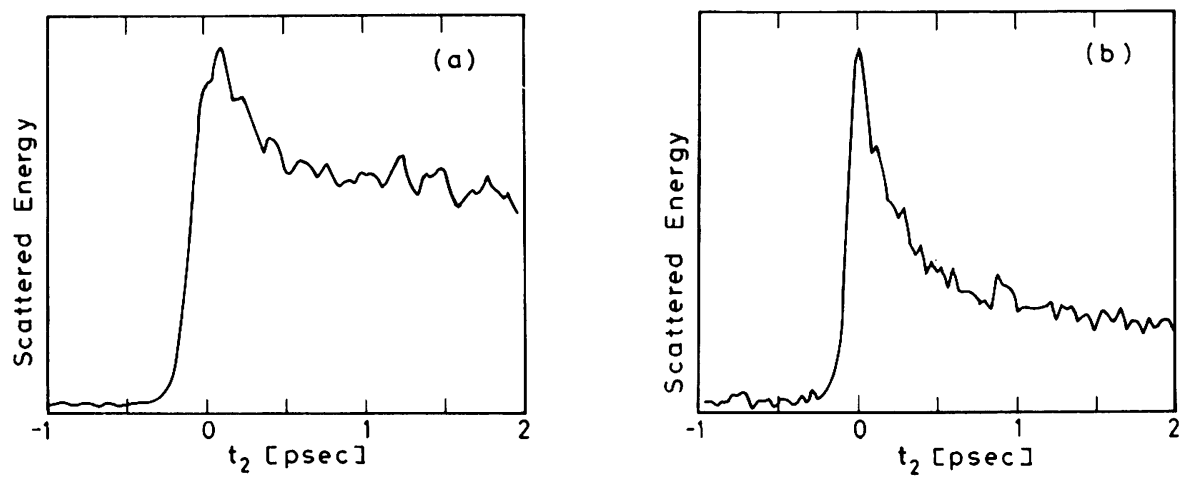

Fig. 6.17. Three-pulse scattering experiment from the organic dye molecules cresyl violet (a) and oxazine 720 (b) at $15 \mathrm{~K}$ using femtosecond light pulses at $620 \mathrm{~nm}$. The scattered energy is plotted as a function of time $t_{2}$ for zero relative delay $t_{1}$. The signal decay with a time constant of several hundred femtoseconds corresponds to the energy redistribution within the first excited electronic states of the dye molecules [6.142] 


\section{b) Measurements of Dephasing Times}

Ippen et al. have extended their experiments to measure the short dephasing times in dye molecules. The dephasing processes were determined by varying the time $t_{1}$, while keeping $t_{2}$ constant at $1.3 \mathrm{ps}$. The signal scattered by the third pulse was detected in two directions $k_{4}=k_{c}+k_{b}-k_{a}$ and $k_{5}=k_{c}+k_{a}-k_{b}$. It has been shown in [6.142] that inhomogeneously broadened transitions with long dephasing times $T_{2}>t_{\mathrm{p}}$ give scattered signal curves which do not peak at time zero.

Figure 6.18 shows the results for cresylviolet in PMMA at $15 \mathrm{~K}$ (Fig. 6.18a) and at $290 \mathrm{~K}$ (Fig. 6.18b). The asymmetry is detected for the low-temperature sample. One observes a $60 \mathrm{fs}$ separation of the peaks of the signal curves which demonstrates the presence of inhomogeneous line broadening. With increasing temperature the splitting of the peaks decreases. At room temperature (see Fig. 6.18b) the coincidence of the two signal curves suggests that homogeneous broadening dominates. The same experiments have been performed with Nile blue and oxazine 720 . For both dyes the scattered curves coincide over the entire temperature range $(15-300 \mathrm{~K})$. These results have been explained as follows: For Nile blue and oxazine 720 , the excitation photons at $620 \mathrm{~nm}$ have energies of several hundred wavenumbers in excess of the $0-0$ transition energy. At large excess energies, background states of high densities exist and a rapid decay of the initially prepared states into these background states can occur. In cresyl violet, on the other hand, low-lying vibronic states, interacting with a small number of background states, were populated; these decay relatively slowly at low temperatures.
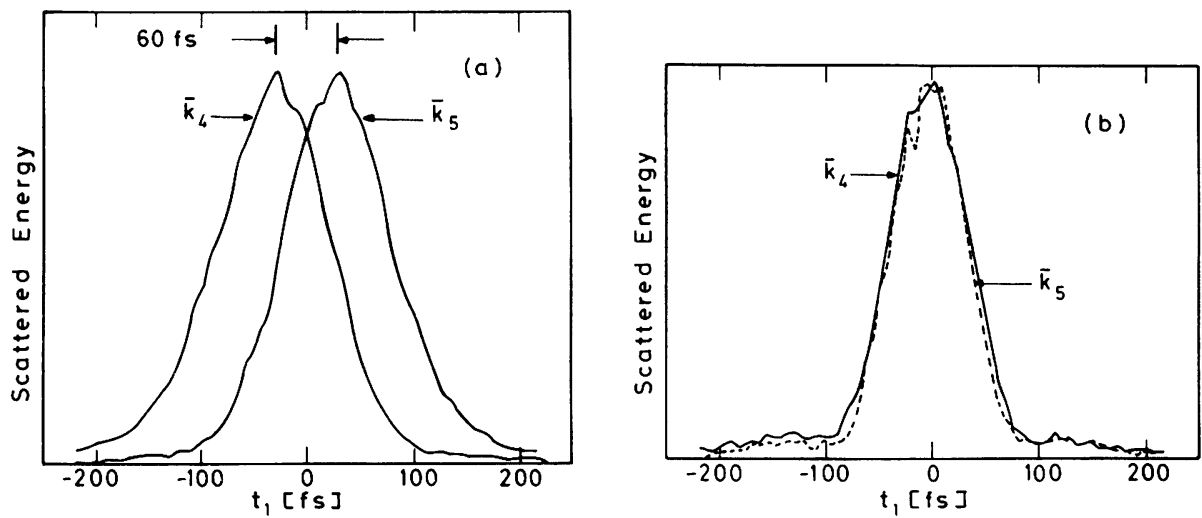

Fig. 6.18a, b. Three-pulse echo experiments for the study of dephasing processes in large dye molecules. For a constant pulse separation, $t_{2}=1.3 \mathrm{ps}$, the first delay time $t_{1}$ was varied and the three-pulse echo energy was detected in the two directions $\boldsymbol{k}_{4}=\boldsymbol{k}_{\mathrm{c}}+\boldsymbol{k}_{\mathrm{b}}-\boldsymbol{k}_{\mathrm{a}}$ and $\boldsymbol{k}_{\mathrm{5}}=\boldsymbol{k}_{\mathrm{c}}+\boldsymbol{k}_{\mathrm{a}}-\boldsymbol{k}_{\mathrm{b}}$. The asymmetry found in (a) - cresyl violet in PMMA at $15 \mathrm{~K}$ - indicates the presence of inhomogeneous line broadening. At higher temperatures the asymmetry disappears. At room temperature (b) the two curves coincide. Here, homogeneous line broadening is dominant [6.142] 

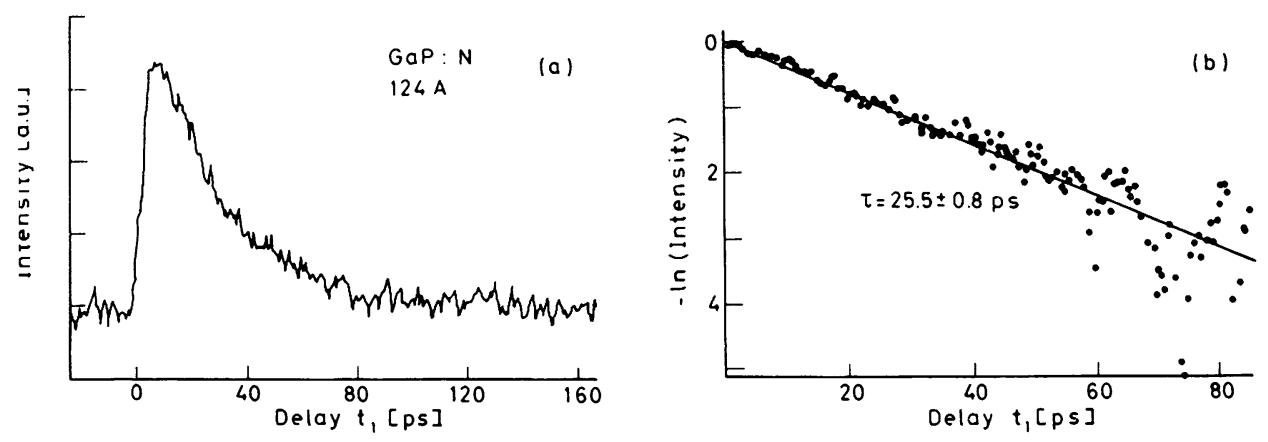

Fig. 6.19a, b. Accumulated photon echo from the nitrogen bond exciton in GaP:N at $1.5 \mathrm{~K}(\lambda=$ $534.95 \mathrm{~nm}$ ). The echo intensity is plotted in a linear scale (a) and in a logarithmic scale (b) versus delay time $t_{1}$. The low-temperature decay time of $25.5 \mathrm{ps}$ is interpreted as the exciton relaxation time out of the initially excited A state [6.131]

\section{c) Accumulated Three-Pulse Echos}

Accumulated three-pulse echos have been observed in a number of molecular crystals and semiconductors where the existence of a long-lived bottleneck state allowed the accumulation of the population grating [6.9,134,136-138]. The experiments discussed here were performed by Wiersma's group [6.131]. The authors investigated nitrogen-doped $\mathrm{GaP}$ with a N-concentration of $1.3 \times$ $10^{16} \mathrm{~cm}^{-3}$. The nitrogen bound exciton at $534.95 \mathrm{~nm}$ was investigated at $1.5 \mathrm{~K}$. In optical absorption only the $A$-state $(J=1)$ can be reached from the ground state $(J=0)$ while the energetically lower $B$-state $(J=2)$ is forbidden and is only seen in emission. The long lifetime of $4 \mu$ s makes the $B$-state a bottleneck state. In the experiment a synchronously pumped picosecond dye laser was used at the high repetition rate of $80 \mathrm{MHz}$ without amplification. The time dependence of the accumulated echo intensity as a function of the delay time $t_{1}$ is plotted on a linear scale and logarithmic scale in Fig. 6.19a and b, respectively. From Fig. $6.19 \mathrm{~b}$, a low-temperature decay time of $25.5 \mathrm{ps}$ was deduced. This decay time is interpreted as the exciton relaxation time from the $A$ - to the $B$-state. The rapid transfer process explains the absence of the $A$-line in the low-temperature emission spectrum. The temperature dependence of the decay times gives two activation energies. It appears that two distinct interaction processes determine the exciton lifetime.

\section{d) Two-Color Echos}

The three-pulse echo proves to be a versatile tool for the study of vibrational deactivation processes when different laser frequencies are applied for the excitation and probing processes. In the upper part of Fig. 6.20 a four-level system (A) is depicted, where the vibrational states $|a\rangle$ and $|b\rangle$ belong to the electronic 


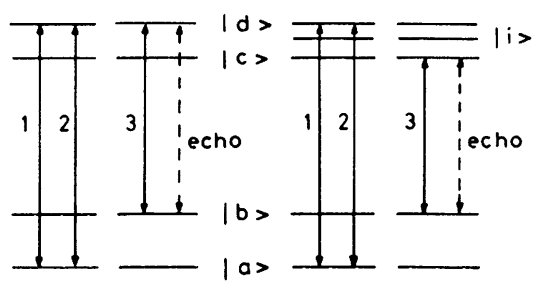

( A )

(B)

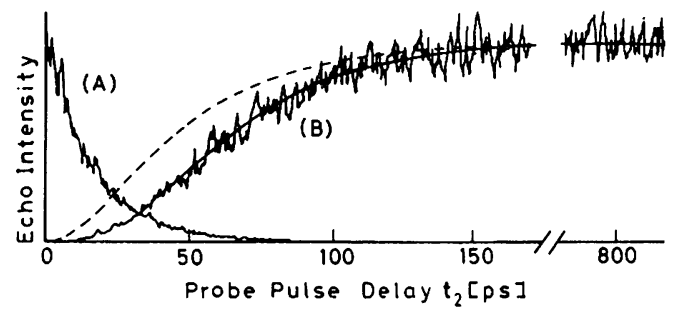

Fig. 6.20. Two-color stimulated photon echos applied to the study of energy relaxation pathways. Left part: Level scheme of the two-color experiment. In case A the first two excitation pulses 1 and 2 generate the population grating in states $|a\rangle$ and $|d\rangle$. Pulses 3 has a wavelength probing only the grating of levels $|b\rangle$ and $|d\rangle$. Varying the delay time $t_{2}$ between pulses 2 and 3 allows one to measure the decay of the population of state $|d\rangle$ (curve A). In case B the wavelength of the probing pulse is selected in order to probe states $|b\rangle$ and $|c\rangle$. An echo found with this probing wavelength allows one to follow the population grating of state $|c\rangle$ or $|b\rangle$ built up in the course of relaxation out of state $|d\rangle$. Curve $\mathrm{B}$ in the right part of the figure shows a delayed build-up of this echo suggesting the existence of additional levels $|i\rangle$ populated during the relaxation process [6.131]

ground state, and $|c\rangle$ and $|d\rangle$ to the excited electronic state. With two excitation pulses 1 and 2, a population grating is generated in the levels $|a\rangle$ and $|d\rangle$; the probe pulse 3 connects levels $|b\rangle$ and $|d\rangle$. When the inhomogeneous broadening in the different levels is correlated, the third pulse generates an echo signal. The echo amplitude measured as a function of the time $t_{2}$ monitors the population decay of state $|d\rangle$. Data from a related experiment are shown in curve $\mathrm{A}$ of the right part of Fig. 6.20. The system pentacene in naphthalene was investigated. The first excitation pulse at $17335 \mathrm{~cm}^{-1}$ prepares a vibrational level at $747 \mathrm{~cm}^{-1}$ above the absorption origin. The probing pulse at $v_{3}=16579 \mathrm{~cm}^{-1}$ monitors the occupation of state $|d\rangle$; curve A may be fitted by an exponential decay with a time constant of $33 \mathrm{ps}$. With the smaller probing frequency $v_{3} / c=15832 \mathrm{~cm}^{-1}$ transitions between levels $|b\rangle$ and $|c\rangle$ are monitored [see Fig. 6.20, scheme (B)]. According to the experimental curve B in the right part of Fig. 6.20 no signal is detected at time zero, i.e. no population is found in the two levels $|b\rangle$ and $|c\rangle$. The echo intensity starts to increase delayed. Within $100 \mathrm{ps}$ it reaches a plateau, where the signal stays constant for the observation time of $800 \mathrm{ps}$ (the lifetime of state $|c\rangle$ is $20 \mathrm{~ns})$. The echo-curve B indicates a population transfer from level $|d\rangle$ to level $|c\rangle$. A more careful inspection of the experimental data suggests a complex transfer process. The experimental echo-curve B deviates from the broken curve which is calculated for a direct population transfer from state $|d\rangle$ to state $|c\rangle$. As a consequence, the authors conclude that there exist intermediate levels $|i\rangle$ with short lifetimes of the order of $20 \mathrm{ps}$ involved in the relaxational processes from level $|d\rangle$ to level $|c\rangle$.

This section clearly demonstrates the power of time-resolved multiple-pulse coherent techniques. The possibility of measuring dephasing times $T_{2}$ for inhomo- 
geneously broadened transitions, and also of studying relaxational path ways is very promising. We mention corresponding experiments in the frequency domain: The techniques of spectral hole-burning have become well established. Holes are first "burnt" in an inhomogeneously broadened transition and the widths are subsequently monitored by properly delayed pulses. From the frequency width, the coherent dephasing time may be determined, while the decay of the holes gives information on the energy relaxation $T_{1}$ due to cross correlation within the inhomogeneously broadened line or due to the energy decay to other levels. Time- and frequency-domain methods are intimately related. Optimum information is gained by a combination of both techniques.

\subsection{Summary}

In this chapter we have discussed more than thirty investigations of time-resolved coherent spectroscopy. Many more experiments may be found in the literature. From the various time-resolved coherent techniques we can obtain the following information:

i) whether a transition is homogeneously broadened,

ii) the dephasing time of homogeneously or inhomogeneously broadened transitions,

iii) dominant line broadening mechanisms,

iv) pure dephasing times,

v) energy relaxation times,

vi) mechanisms and pathways of energy relaxation,

vii) collision times,

viii) nonresonant susceptibilities,

ix) transition frequencies within congested spectral regions,

$\mathrm{x})$ precise frequency differences of vibrational modes separated by up to $10 \mathrm{THz}$.

\subsection{Appendix}

For the analytical solution of $(6.3-5)$ we introduced in Sect. 6.1 .2 the vectorial form $\boldsymbol{\rho}(t)$ of the density matrix and the transformation matrices $\underline{X}(A)$ and $\underline{Y}(t)$ for the excitation process and the free-evolution process, respectively. In detail they are given as follows:

$$
\rho_{1}(t)=\rho_{b b} ; \quad \rho_{2}(t)=\rho_{b a} ; \quad \rho_{3}(t)=\rho_{a b} ; \quad \rho_{4}(t)=\rho_{a a}
$$




$$
\begin{aligned}
& Y(t)=\left(\begin{array}{cccc}
1 & 0 & 0 & Y_{14} \\
0 & \mathrm{e}^{\left(\mathrm{i} \Delta-1 / T_{2}\right) t} & 0 & 0 \\
0 & 0 & \mathrm{e}^{\left(-\mathrm{i} \Delta-1 / T_{2}\right) t} & 0 \\
0 & 0 & 0 & \mathrm{e}^{-t / T_{1}}
\end{array}\right) \\
& X\left(A_{j}\right)=\frac{1}{2}\left(\begin{array}{cccc}
1+\cos A_{j} & -\mathrm{i} \sin A_{j} \mathrm{e}^{\mathrm{i} k_{j} r} & \mathrm{i} \sin A_{j} \mathrm{e}^{-\mathrm{i} k_{j} r} & 1-\cos A_{j} \\
-\mathrm{i} \sin A_{j} \mathrm{e}^{-\mathrm{i} k_{j} r} & 1+\cos A_{j} & \left(1-\cos A_{j}\right) \mathrm{e}^{-2 \mathrm{i} k_{j} r} & \mathrm{i} \sin A_{j} \mathrm{e}^{-\mathrm{i} k_{j} r} \\
\mathrm{i} \sin A_{j} \mathrm{e}^{\mathrm{i} k_{j} r} & \left(1-\cos A_{j}\right) \mathrm{e}^{2 \mathrm{i} k_{j} r} & 1+\cos A_{j} & -\mathrm{i} \sin A_{j} \mathrm{e}^{\mathrm{i} k_{j} r} \\
1-\cos A_{j} & \mathrm{i} \sin A_{j} \mathrm{e}^{\mathrm{i} k_{j} r} & -\mathrm{i} \sin A_{j} \mathrm{e}^{-\mathrm{i} k_{j} r} & 1+\cos A_{j}
\end{array}\right)
\end{aligned}
$$

where $A_{j}$ is the pulse area of the excitation pulse, $k_{j}$ is the wave vector. The matrix element $Y_{14}$ depends on the type of two-level system used. When a closed two-level system is treated, where the system is only in level $|a\rangle$ or $|b\rangle, Y_{14}$ becomes $Y_{14}=1-\exp \left(-t / T_{1}\right)$. For an open system, where level $|a\rangle$ decays into a long-lived state $|c\rangle, Y_{14}$ vanishes: $Y_{14}=0$.

\section{References}

6.1 J.A. Giordmaine, W. Kaiser: Phys. Rev. 144, 676 (1966)

6.2 S.F. Fischer, A. Laubereau: Chem. Phys. Lett. 35, 6 (1975)

6.3 D.W. Oxtoby: Adv. Chem. Phys. 40, 1 (1979)

6.4 R. Kubo: In Fluctuations, Relaxation and Resonance in Magnetic Systems, ed. by D. ter Haar (Plenum, New York 1962) p. 101

6.5 R.G. Gordon: Adv. Magn. Reson. 3, 1 (1968)

6.6 R.G. Gordon: J. Chem. Phys. 39, 2788 (1963)

6.7 A. Laubereau, W. Kaiser: Rev. Mod. Phys. 50, 607 (1978)

6.8 A. Penzkofer, A. Laubereau, W. Kaiser: Progr. Quant. Electron. 6, 55 (1979)

6.9 W.H. Hesselink, D.A. Wiersma: J. Chem. Phys. 73, 648 (1980)

6.10 A. Yariv: Quantum Electronics (Wiley, New York 1975) p. 372

6.11 N.A. Kurnit, I.D. Abella, S.R. Hartmann: Phys. Rev. Lett. 13, 567 (1964)

6.12 I.D. Abella, N.A. Kurnit, S.R. Hartmann: Phys. Rev. 141, 391 (1966)

6.13 S.R. Hartmann: IEEE J. Quant. Electron. QE-4, 802 (1968)

6.14 R.F. Loring, S. Mukamel: Chem. Phys. Lett. 114, 426 (1985)

6.15 R.G. Gordon: J. Chem. Phys. 43, 1307 (1965)

6.16 R.G. Gordon: J. Chem. Phys. 44, 1830 (1966)

6.17 G. Placzek: Handbuch der Radiologie, ed. by E. Marx (Akad. Verlagsges., Leipzig 1934) p. 1

6.18 N. Kohles, A. Laubereau: Appl. Phys. B 39, 141 (1986)

6.19 B. Dick: Chem. Phys. 113, 131 (1987)

6.20 N. Bloembergen: Am J. Phys. 35, 989 (1967)

6.21 W. Kaiser, M. Maier: In Laser Handbook, Vol. 2, ed. by F.T. Arecchi, E.O. Schulz-Dubois (North-Holland, Amsterdam 1972) p. 1077

6.22 S.A. Akhmanov: Mater. Res. Bull. 4, 455 (1969)

6.23 R.L. Carman, F. Shimizu, C.S. Wang, N. Bloembergen: Phys. Rev. A 2, 60 (1970)

6.24 M. Maier, W. Kaiser, J.A. Giordmaine: Phys. Rev. 177, 580 (1969) 
Y.R. Shen, N. Bloembergen: Phys. Rev. 137A, 1786 (1965)

N. Bloembergen: Nonlinear Optics (Benjamin, New York 1965)

P.D. Maker, R.W. Terhune: Phys. Rev. 137A, 801 (1965)

A. Penzkofer, W. Kaiser: Opt. Quant. Electron. 9, 315 (1977)

D. von der Linde, A. Laubereau, W. Kaiser: Phys. Rev. Lett. 26, 954 (1971)

R.R. Alfano, S.L. Shapiro: Phys. Rev. Lett. 26, 1247 (1971)

C.H. Lee, D. Richard: Appl. Phys. Lett. 32, 168 (1978)

J.P. Heritage: Appl. Phys. Lett. 34, 470 (1979)

J. Kuhl, D. von der Linde: In Ultrafast Phenomena III, Proc. Topical Meeting, GarmischPartenkirchen, Germany, June 1982, Springer Ser. Chem. Phys., Vol. 23, ed. by K.B. Eisenthal, R.M. Hochstrasser, W. Kaiser (Springer, Berlin, Heidelberg 1982) p. 201

R. Leonhardt, W. Holzapfel, W. Zinth, W. Kaiser: Chem. Phys. Lett. 133, 373 (1987)

A. Laubereau: Chem. Phys. Lett. 27, 600 (1974)

A. Laubereau, G. Wochner, W. Kaiser: Opt. Commun. 17, 91 (1976)

W. Zinth, H.J. Polland, A. Laubereau, W. Kaiser: Appl. Phys. B 26, 77 (1981)

H. Graener, A. Laubereau, J.W. Nibler: Opt. Lett. 9, 165 (1984)

M.L. Geirnaert, G.M. Gale, C. Flytzanis: Phys. Rev. Lett. 52, 815 (1984)

M.L. Geirnaert, G.M. Gale: Chem. Phys. 86, 205 (1984)

J. Chesnoy: Chem. Phys. Lett. 125, 267 (1986)

G.M. Gale, P. Guyot-Sionnest, W.Q. Zheng: Opt. Commun. 58, 395 (1986)

M. van Exter, A. Lagendijk: Opt. Commun. 56, 191 (1985)

W. Zinth, R. Leonhardt, W. Holzapfel, W. Kaiser: J. Quant. Electron. QE-24, 455 (1988)

R. Leonhardt, W. Holzapfel, W. Zinth, W. Kaiser: private communication

D.W. Oxtoby, D. Levesque, J.J. Weis: J. Chem. Phys. 68, 5528 (1978)

J.P. Riehl, D.J. Diestler: J. Chem. Phys. 64, 2593 (1976)

D.J. Diestler, R.S. Wilson: J. Chem. Phys. 62, 1572 (1975)

P.A. Madden, R.M. Lynden-Bell: Chem. Phys. Lett. 38, 163 (1976)

R.M. Lynden-Bell, G.C. Tabisz: Chem. Phys. Lett. 46, 175 (1977)

S. Bratos, E. Marechal: Phys. Rev. A 4, 1078 (1971)

W.G. Rothschild: J. Chem. Phys. 65, 455 (1976)

W.G. Rothschild: J. Chem. Phys. 65, 2958 (1976)

D.J. Diestler, J. Manz: Mol. Phys. 33, 227 (1977)

R. Wertheimer: Mol. Phys. 35, 257 (1978)

R. Wertheimer: Chem. Phys. Lett. 52, 224 (1977)

D. Oxtoby: J. Chem. Phys. 70, 2605 (1979)

R.M. Shelby, C.B. Harris, P.A. Cornelius: J. Chem. Phys. 70, 34 (1979)

S.F. Fischer, A. Laubereau: Chem. Phys. Lett. 55, 189 (1978)

S. Mukamel: Phys. Rev. A 26, 617 (1982)

S. Mukamel: Phys. Rev. A 28, 3480 (1983)

W.G. Rothschild, M. Perrot, F. Guillaume: Chem. Phys. Lett. 128, 591 (1986)

W. Zinth, A. Laubereau, W. Kaiser: Opt. Commun. 26, 457 (1978)

F.M. Kamga, M.G. Sceats: Opt. Lett. 5, 126 (1980)

M.G. Sceats, F. Kamga, D. Podolski: In Picosecond Phenomena II, Proc. Topical Meeting, Cape Cod, Mass., USA, June 1980, Springer Ser. Chem. Phys., Vol. 14, ed. by R.M. Hochstrasser, W. Kaiser, C.V. Shank (Springer, Berlin, Heidelberg 1980) p. 348

W. Zinth: Opt. Commun. 34, 479 (1980)

B.K. Rhee, W.E. Bron, J. Kuhl: Phys. Rev. B 30, 7358 (1984)

S. Velsko, J. Trout, R.M. Hochstrasser: J. Chem. Phys. 79, 2114 (1983)

D.E. McCumber, M.D. Sturge: J. Appl. Phys. 34, 1682 (1963)

R. Orbach: IEEE Trans. Sonics Ultrason. SU14, 140 (1967)

R. Orbach, L.A. Vredevoe: Physics 1, 91 (1964)

P.G. Klemens: Phys. Rev. 148, 845 (1966) 
6.71 I.I. Abram, R.M. Hochstrasser: J. Chem. Phys. 72, 3617 (1980)

6.72 S. Velsko, R.M. Hochstrasser: J. Chem. Phys. 82, 2180 (1985)

6.73 G.J. Small: Chem. Phys. Lett. 57, 501 (1978)

6.74 K.E. Jones, A.H. Zewail: In Advances in Laser Chemistry, ed. by A.H. Zewail (Springer, New York 1978) p. 196

6.75 C.B. Harris, R.M. Shelby, P.A. Cornelius: Phys. Rev. Lett. 38, 1415 (1977)

6.76 C.B. Harris J. Chem. Phys. 67, 5607 (1977)

6.77 P. de Bree, D.A. Wiersma: J. Chem. Phys. 70, 790 (1978)

6.78 R.G. Delle Valle, P.F. Fracassi, R. Righini, S. Califano: Chem. Phys. 74, 179 (1983)

6.79 D.D. Dlott: Ann. Rev. Phys. Chem. 37, 157 (1986)

6.80 W.H. Hesselink, D.A. Wiersma: Chem. Phys. Lett. 65, 300 (1979)

6.81 D. von der Linde, J. Kuhl, H. Klingenberg: Phys. Rev. Lett. 44, 1505 (1980)

6.82 A. Laubereau, D. von der Linde, W. Kaiser: Phys. Rev. Lett. 27, 802 (1971)

6.83a W.E. Bron, J. Kuhl, B.K. Rhee: Phys. Rev. B 34, 6961 (1986)

6.83b J. Kuhl, W.E. Bron: Solid State Commun. 49, 935 (1984)

6.84 G.M. Gale, A. Laubereau: Opt. Commun. 44, 273 (1983)

6.85 A. Laubereau, G. Wochner, W. Kaiser: Opt. Commun. 14, 75 (1975)

6.86 A. Laubereau: In Semiconductors Probed by Ultrafast Laser Spectroscopy, Vol. 1, ed. by R.R. Alfano (Academic, Orlando 1984) p. 275

6.87 G.M. Gale, F. Vallee, C. Flytzanis: Phys. Rev. Lett. 57, 1867 (1986)

6.88 G.M. Gale, P. Guyot-Sionnest, W.Q. Zheng, C. Flytzanis: Phys. Rev. Lett. 54, 823 (1985)

6.89 G.M. Gale, F. Vallee, C. Flytzanis: In Time Resolved Vibrational Spectroscopy, Proc. Conf. June 1985, Springer Proc. Phys. IV, ed. by A. Laubereau, M. Stockburger (Springer, Berlin, Heidelberg 1985) p. 117

6.90 C. Flytzanis, G.M. Gale, M.L. Geirnaert: In Applications of Picosecond Spectroscopy to Chemistry, ed. by K.B. Eisenthal (Reidel, Amsterdam 1984) p. 205

6.91 P.L. Decola, R.M. Hochstrasser, H.P. Frommsdorff: Chem. Phys. Lett. 72, 1 (1980)

6.92 B.H. Hesp. D.A. Wiersma: Chem. Phys. Lett. 75, 423 (1980)

6.93 K. Duppen, B.H. Hesp, D.A. Wiersma: Chem. Phys. Lett. 79, 399 (1981)

6.94 K. Duppen, D.P. Weitekamp, D.A. Wiersma: J. Chem. Phys. 79, 5835 (1983)

6.95 C.L. Schosser, D.D. Dlott: J. Chem. Phys. 80, 1394 (1984)

6.96 F. Ho, W.S. Tsay, J. Trout, R.M. Hochstrasser: Chem. Phys. Lett. 83, 5 (1981)

6.97 F. Ho, W.S. Tsay, S. Velsko, R.M. Hochstrasser: Chem. Phys. Lett. 97, 141 (1983)

6.98 S. Velsko, J. Trout, R.M. Hochstrasser, J. Chem. Phys. 79, 2114 (1983)

6.99 T.J. Trout, S. Velsko, R. Bozio, P.L. Decola, R.M. Hochstrasser: J. Chem. Phys. 81, 4746 (1984)

6.100 S. Velsko, R.M. Hochstrasser: J. Chem. Phys. 89, 2240 (1985)

6.101 R.M. Hochstrasser: In Time Resolved Vibrational Spectroscopy, Proc. Conf. June 1985, Springer Proc. Phys. IV, ed. by A. Laubereau, M. Stockburger (Springer, Berlin, Heidelberg 1985) p. 96

6.102 T.J. Kosic, R.E. Cline Jr., D.D. Dlott: J. Chem. Phys. 81, 4932 (1984)

6.103 R.F. Loring, S. Mukamel: In Time Resolved Vibrational Spectroscopy, Proc. Conf. June 1985, Springer Proc. Phys. IV, ed. by A. Laubereau, M. Stockburger (Springer, Berlin, Heidelberg 1985) p. 293

6.104 H.R. Telle, A. Laubereau: Chem. Phys. Lett. 94, 467 (1983)

6.105 W. Zinth, W. Kaiser: In Organic Molecular Aggregates, Springer Ser. Solid-State Sci., Vol. 49, ed. by P. Reinecker, H. Haken, H.C. Wolf (Springer, Berlin, Heidelberg 1983) p. 124

6.106 R. Leonhardt, W. Holzapfel, W. Zinth, W. Kaiser: private communication

6.107a H. Graener, A. Laubereau: Opt. Commun. 54, 141 (1985)

6.107b H. Graener, A. Laubereau: In Time Resolved Vibrational Spectroscopy, Proc. Conf. June 1985, Springer Proc. Phys. IV, ed. by A. Laubereau, M. Stockburger (Springer, Berlin, Heidelberg 1985) p. 11 
6.108 S. De Silvestri, J.G. Fujimoto, E.P. Ippen, E.B. Gamble Jr., L.R. Williams, K.A. Nelson: Chem. Phys. Lett. 116, 146 (1985)

6.109 A. Laubereau, G. Wochner, W. Kaiser: Chem. Phys. 28, 363 (1978)

6.110 A. Laubereau, G. Wochner, W. Kaiser: Phys. Rev. A 13, 2212 (1976)

6.111 C.B. Harris, H. Auweter, S.M. George: Phys. Rev. Lett. 44, 737 (1980)

6.112 S.M. George, H. Auweter, C.B. Harris: J. Chem. Phys. 73, 5573 (1980)

6.113 S.M. George, C.B. Harris: Phys. Rev. A 28, 863 (1983)

6.114 S.M. George, A.L. Harris, M. Berg, C.B. Harris: J. Chem. Phys. 80, 83 (1984)

6.115 J.C. Diels: IEEE J. Quant. Electron. QE-16, 1020 (1980)

6.116 R.F. Loring, S. Mukamel: J. Chem. Phys. 83, 2116 (1985)

6.117 W. Zinth, W. Kaiser: In Lecture Notes in Physics, Vol. 182, ed. by J.D. Harvey, D.F. Walls (Springer, New York 1983) p. 152

6.118 W. Zinth, M.C. Nuss, W. Kaiser: Chem. Phys. Lett. 88, 257 (1982)

6.119 W. Zinth, M.C. Nuss, W. Kaiser: Opt. Commun. 44, 262 (1983)

6.120 W. Zinth, M.C. Nuss, W. Kaiser: Phys. Rev. A 30, 1139 (1984)

6.121 M.C. Nuss, W. Zinth, W. Kaiser: J. Opt. Soc. Am. B 2, 322 (1985)

6.122 M.A. Collins, P.A. Madden, A.D. Buckingham: Chem. Phys. 94, 291 (1985)

6.123 M.D. Crisp: Phys. Rev. A 1, 1604 (1970)

6.124 H.J. Hartmann, A. Laubereau: Opt. Commun. 47, 117 (1983)

6.125 H.J. Hartmann, A. Laubereau: J. Chem. Phys. 80, 4663 (1984)

6.126 H.J. Hartmann, K. Bratengeier, A. Laubereau: Chem. Phys. Lett. 108, 555 (1984)

6.127 H.J. Hartmann, H. Schleicher, A. Laubereau: Chem. Phys. Lett. 116, 392 (1985)

6.128 D.W. Phillion, D.J. Kuizenga, A.E. Siegman: Appl. Phys. Lett. 27, 85 (1975)

6.129 A.E. Siegman: Appl. Phys. Lett. 30, 21 (1977)

6.130 R. Trebino, A.E. Siegman: J. Chem. Phys. 79, 3621 (1983)

6.131 K. Duppen, L.W. Molenkamp, D.A. Wiersma: Physica 127B, 349 (1984)

6.132 L. Schultheis, M.D. Sturge, J. Hegarty: Appl. Phys. Lett. 47, 995 (1985)

6.133 W.H. Hesselink, D.A. Wiersma: Chem. Phys. Lett. 56, 227 (1978)

6.134 W.H. Hesselink, D.A. Wiersma: Phys. Rev. Lett. 43, 1991 (1979)

6.135 W.H. Hesselink, D.A. Wiersma: J. Chem. Phys. 74, 886 (1981)

6.136 L.W. Molenkamp, D.A. Wiersma: J. Chem. Phys. 80, 3054 (1984)

6.137 K. Duppen, D.P. Weitekamp, D.A. Wiersma: Chem. Phys. Lett. 108, 551 (1984)

6.138 K. Duppen, D.P. Weitekamp, D.A. Wiersma: Chem. Phys. Lett. 106, 147 (1984)

6.139 L.W. Molenkamp, D.P. Weitekamp, D.A. Wiersma: Chem. Phys. Lett. 99, 382 (1983)

6.140 A.M. Weiner, E.P. Ippen: Opt. Lett. 9, 53 (1984)

6.141 S. De Silvestri, A.M. Weiner, J.G. Fujimoto, E.P. Ippen: Chem. Phys. Lett. 112, 195 (1984)

6.142 A.M. Weiner, S. De Silvestri, E.P. Ippen: J. Opt. Soc. Am. B 2, 654 (1985)

6.143 J.L. Skinner, H.C. Andersen, M.D. Fayer: J. Chem. Phys. 75, 3195 (1981)

6.144 W.H. Hesselink, D.A. Wiersma, J. Chem. Phys. 75, 4192 (1981)

6.145 D.P. Weitekamp, K. Duppen, D.A. Wierma: Phys. Rev. A 27, 3089 (1983)

6.146 H. Paerschke, K.E. Süsse, B. Wilhelmi: Opt. Quant. Electron. 15, 41 (1983)

6.147 J.G. Fujimoto, T.K. Yee: Appl. Phys. B 34, 55 (1984)

6.148 L. Schultheis, A. Honold, J. Kuhl, K. Köhler, C.W. Tu: Phys. Rev. B 34, 9027 (1986)

6.149 A.M. Weiner, E.P. Ippen: Chem. Phys. Lett. 114, 456 (1985) 


\section{Subject Index}

Aberration correction 67

Absorption 314

- grating 66

- saturation 283

- spectra of electron-hole plasmas 131

Accumulated echo 268, 271

Acetone 247

Acetonitrile 247

Acetylene $\left(\mathrm{C}_{2} \mathrm{H}_{2}\right) \quad 286$

Acoustic phonon 86

Acousto-optic mode-locking 14

Active mode-locking 7

Adiabatic photodissociation 338

ADP (ammonium dihydrogen phosphate)

$40,41,44,50,52$

Adsorbates 95

Ag (silver) 94, 95

$\mathrm{AgBr} 91$

$\mathrm{AgCl} 94$

$\mathrm{AgGaS}_{2} \quad 46$

Air/liquid interface 96

Air/solid interface 96

$\mathrm{Au}$ (gold) 94

Aluminium 176

Amino acids 254

Amorphization 161

Amplification 19

Angle tuning 47, 52

Anharmonic coupling 292, 311

- intramolecular 290

Anharmonic intramolecular interactions 286

Anharmonic mixing 291

Anharmonicities of potential 286

Anisotropic Raman tensor 243

Anisotropy of Raman polarization 81

Antenna energy transfer 390

Approximate phase-matching 37

Aqueous solution 65

Artificial nonlinearity 92

Association of alcohol molecules 290

Autocorrelation function 89

Autocorrelation measurements 56

Automatic phase-matching $65,72,77$ 7-azaindole dimer 348

Azulene 300

Backward emission 73

Bacterial reaction centers 384

Bacteriorhodopsin 396

Bandwidth product 56

Barium sodium niobate $\left(\mathrm{Ba}_{2} \mathrm{NaNb}_{3} \mathrm{O}_{15}\right)$

49

Barium vapor 80

Bathorhodopsin 401

Beam divergence 38

Benzene alcohol 97, 253, 258

Benzophenon 330

1.1'-binaphthyl 323

Binary collisions 248

Biradicals 339

Bistability 91

BK-7 glass 91

Bleaching dynamics of organic dyes 314

Bleaching sequence 396

Boltzmann factor 302

Borosilicate glass 69

Bound exciton 93

Bragg scattering 84,96

Brilliant green 87

Buffer gas 80

Cadmium 62

Cadmium sulfide $91,93,116,134$

Calcite 63, 149

Carbenes 333, 334

Carrier thermalization 137

CARS 63, 66, 250

Cascade process 42

$\mathrm{CCl}_{4} \quad 249,257,260$

$\mathrm{CDA}\left(\mathrm{CsH}_{2} \mathrm{AsO}_{4}\right) \quad 41$

CdS 91, 93, 116, 134

CdSe 134

Cesium vapor 80

$\mathrm{CH}_{2} \mathrm{BrCl} 256$

$\mathrm{CH}_{3} \mathrm{CCl}_{3} 81$

$\mathrm{CH}_{3} \mathrm{I} \quad 82$

Charge density probing 219 
Charge transfer 329

Charged particle emission 172

Chemical intermediates 329

Chirp 25

Chlorobenzene 66

Cholesteric liquid crystals 61

$\mathrm{CO}_{2} \quad 158$

Coherence peak 67,88

Coherence time $t_{\mathrm{c}} \quad 89$

Coherent anti-Stokes Raman probing 59

Coherent anti-Stokes Raman scattering

see CARS 63, 66, 250

Coherent artifact 138

Coherent spectroscopy 253

- in solids 251

Collision time 255

Collision-induced transitions 290

Color center laser 22

Composite materials 97

Continuum generation 90,98

Conversion efficiency $38,40,49,78$

Coumarin 96, 294, 298

CPM laser 16,30

Cresyl violet 269

Cross-correlation curve 285

Crown glass K 897

Crystal damage 53

$\mathrm{CS}_{2}$ 66, 67, 68 (Fig. 3.12c), 72, 97, 157

$\mathrm{CuCl} 116$

Cyanine dyes 61

Cyclohexane $\mathrm{C}_{6} \mathrm{H}_{12} \quad 249$

$\mathrm{D}_{2} 98$

$\mathrm{D}_{2} \mathrm{O} \quad 44,70,90$

Degenerate four-wave mixing (DFWM) 17, 64, 66, 70, 72, 97

Degenerate parametric amplification 41

Density of states 289, 294

Dephasing in liquids 247

Dephasing time $T_{2} \quad 75,238$

Depopulation lifetimes see Energy

relaxation time $T_{1} \quad 310,313$

Diamond 149,252

Dielectric breakdown 54,62

Difference-frequency generation 45

Difference-frequency resonance 69

Diode laser 24

Distributed feedback laser 23,88

DODCI 71

Double proton transfer 348

Double resonance of nonlinear susceptibility 74

Down conversion $45,47,54,56$

Electric dipole transition 241

Electrical isolation 213
Electrical transmission lines 209

Electron donor-acceptor complexes 332

Electron transfer to quinone 389

Electron-hole scattering 190

Electronic autocorrelation measurement 203

Electro-optic Cherenkov radiation 208

Electro-optic wafer prober 218

Energy decay law 307

Energy dissipation to surroundings 296

Energy equalization 284, 309, 313

Energy exchange 292, 294

Energy relaxation time $T_{1} \quad 236,238,268$, 269, 306

Energy transfer in chlorophylls 390

Energy transfer law 305

Energy transfer to translation 287

Excess population 281

Excited-state proton transfer 341

Factor group 253

Far infrared 45, 52, 53

- applications 221

- generation 46, 47

Femtosecond continuum 51,91

Femtosecond dye laser 17

Fermi resonance 158,290

- mixing 292

Fifth harmonic generation 62

Fifth-order correlation function 65

Flow of vibrational energy 286

Fluorescence 368, 404

- excitation spectrum 299

Fourier-transform Raman spectroscopy 259

Four-photon mixing process 69

Fourth harmonic 43

Four-wave difference frequency 63

Four-wave mixing $64,68,83,90,98$

Four-wave up-conversion 62

Frequency mixing 43

Frequency shift 90

Frozen-wave generator 222

Fuchsin 61

Fused silica 70

GaAs $91,117,131,153,161,175,266$

Gain coefficient 75

Gain of electron-hole plasmas 131

Gain factor 47

Gain measurements 314

Gain saturation 76

GaP 150, 251, 252, 271

Gaussian beam 38

Ge (germanium) $87,94,161$ 
Germinate reactions with $\mathrm{CO}_{2}, \mathrm{NO}$, and $\mathrm{O}_{2}$ 374

Giant-pulse lasers 10

Green plant reaction centers

Group delay $38,41,42,77$

395

Group velocity 116,117

- dispersion 38

$\mathrm{H}_{2} \mathrm{O} \quad 90$

$\mathrm{HCl} 263$

Halorhodopsin 409

Heat conduction 308

Heat pipe $61,63,80$

Helical structure 61

Heme proteins $\quad 357-380$

Hexafluoroisopropanol 61

Higher-order cross correlation 59

Higher-order scattering 86

High-power photoconductors 226

High-speed photodetectors 214

High-speed transistors 215

Homogeneously broadened transition 265

Hot-phonon effects 137

Hybrid mode-locking 18

Hydrogen atom transfer 330

Hydrogen gas 77

Hysteresis 92, 96

$\mathrm{I}_{2}$ (iodine) 328

Image reconstruction 72

Impulsive stimulated Brillouin scattering 86

Impulsive stimulated scattering 78

Incoherent phonons 152

Indigo 345

Induced absorption spectra 119

Induced birefringence 67

Induced dichroism 314

Induced population grating 68,83

Infrared bleaching 283

Infrared continuum 55

Infrared spectra 367

InGaAs 136

Inhomogeneous broadening 239, 259

Inhomogeneous line 265

Integrated circuits $217,218,219$

Integrated photoconductors 220

Intensity-and-phase autocorrelator 57

Intensity autocorrelation function 56,59

Intensity grating 85

Interaction Hamiltonian 236

Interaction length 37,38

Interfaces 94
Interferometric autocorrelation 57,58

(Fig. 3.9a)

Interferometric technique 67

Intermolecular energy transfer 303,304 , 305, 306

Intermolecular proton transfer 342

Intermolecular relaxation of energy 295

Intermolecular vibronic relaxation 315

Internal conversion 281,300

Internal temperature $299,300,301$

Intervalley scattering 155

Intramolecular energy transfer 286, 290

Intramolecular proton transfer 343

Inverse gap effect 335

Inversion symmetry 37,53

Isoenergetic combination modes 302

Isolated binary collisions 308

KB 5 (potassium penta borate) 44

KDP (potassium dihydrogen phosphate)

$38,39,40,41,43,51,53$

KD*P 40,44

Kerr cell 227

Kerr nonlinearity 26

Kramers model 321

$\mathrm{KTiOPO}_{4}(\mathrm{KTP}) \quad 40$

Langevin equation 322

Langmuir-Blodgett film 95

Laser heating 94

Laser-induced breakdown 54

Laser-induced heating 377

Laser-induced orientational grating 87

Lattice temperature 172

Lattice vibrations 209

Light harvesting 390

Light-induced grating 64

Light-induced phase-shift 68

$\mathrm{LiIO}_{3} 40,41,44,45,46,51,53$

$\mathrm{LiNbO}_{3} 38,39,41,47,49,50,51$

Local temperature 309

Luminescence 55

Macroscopic heat conduction 311

Melting 94

Metal colloids 98

Metal vapor 61,78

Methanol 59, 96

Microcanonical distribution 301

Microwave applications 221

Microwave generation 222

Mid-infrared 52, 53

Millimeter wave applications 221

Model for energy transfer 306

Models for primary processes 399 
Mode-locked semiconductor 24

Mode-locking 5

- of color center 22

Molecular beam 324, 349

Molecular crystals 152

Molecular dynamics simulations 372

Molecular thermometer 309

Monolayer 95

Mott transition 135

Multiphonon states 156

Multiphoton processes 404

Multiple excitation processes 264

Multiple quantum well 93

Multiple trapping 144

$\mathrm{NaCl} 70$

$\mathrm{Ne}$ (neon) 62

Nearly degenerate four-wave mixing 65 , 66,73

Nitrenes 337

Nitrobenzene 66

Nitrogen 256

Noble gases 61

Non-centrosymmetric crystals 42

Noncritical phase-matching 51

Nondegenerate four-wave mixing 65

Nonexponential dephasing 82

Noninvasive probing 212

Nonlinear Kerr media 90

Nonlinear polarization 37

Nonlinear refraction 96

Nonlinear refractive index $66,67,91,92$, 96, 100

Nonlinear Schrödinger equation 99,100

Nonlinear susceptibility $\chi^{(3)} \quad 60,94$

Nonlinear transmission lines 210

Nonlinear wave equation 85,99

Nonresonant susceptibility $\chi_{\mathrm{NR}}^{(3)} \quad 246,249$

NOR gate 93

Off-axis geometry 43,67

One-photon resonance $69,70,72$

Optical absorption 401,404

Optical bistability 92, 96

Optical inhomogeneity 50

Optical Kerr effect 97

Optical parametric amplifier (OPA) 48, 50

Optical parametric oscillator (OPO) 47 , 48

Optical phase conjugation $67,69,70,73$

Optical phonon lifetimes 149

Optical pulse compression 59

Optical rectification 187, 207, 222

Optical spectra 359

Optical Stark effect 93
Optical surface spectroscopy 95

Optoelectronic devices 185

Optoelectronic measurement systems 211

Organic polymers 72

Orientational Kerr effect 66,67

Orientational relaxation 141

Oxazine $720 \quad 269$

Parametric continuum 53

Parametric emission 65

Parametric fluorescence 68

Parametric light gate 54

Parametric oscillation $51,70,71$

Parametric process 285

Parametric superluminescence 48

Passive mode-locking $9,11,15$

Pentacene in naphthalene 266

Pentadecanoic acid 96

Peptides 254

Phase conjugation 87

Phase-grating 67

Phase-matching $42,61,99,246$

Phase-matching bandwidth $\Delta v \quad 39,44$

Phase memory 86

Phase mismatch 37

Phase modulation 81, 223

Phase relaxation time $T_{2} \quad 236,237,247$, 251, 267, 270

Phenol 95

Phonon dynamics 146

Photochemistry 395

Photoconducting antenna 224

Photoconducting electrical pulse generators 200

Photoconducting electronic sampling 202

Photoconducting materials 188

Photoconductive switches 221, 227

Photocycle 397

Photodissociation of $\mathrm{I}_{2} \quad 328$

Photoelectric emission 217

Photo-emissive sampling 217

Photo-excited carrier distribution 139

Photofragmentation 349

Photoisomerization 320, 325

Photon counting 54

Photon echo 240, 265

Photophysics 375

Photoreduction 330

Photosynthesis 374, 380-395

Phycobilin energy transfer 393

Picosecond continuum 44, 53

Picosecond mass spectrometry 349

Picosecond photoconductors 198

Plasma density 172

Plasma frequency 170 
Pockel's cell 227

Polarization 36

- mismatch scheme 43

Polydiacetylene 72, 85, 97

Population lifetime $T_{1}$ see also Energy

relaxation time $75,279,285,288,289$,

290, 291, 295, 296, 306, 311

Population probability of vibrational states 302

Proustite $\left(\mathrm{Ag}_{3} \mathrm{AsS}_{3}\right) \quad 53$

Pulse area 238, 243, 244

Pulse compression 25, 93

Pulse dispersion 210

Pulse shape analysis 56

Pump-probe coupling 88

Pump-probe experiment 88

Pure dephasing time $T_{2}^{*} \quad 238$

Pure dephasing time in solids 252

Purple membrane pigments 404

Pyridine 95, 261

Quantum beats 256

Quantum-confined Stark effect 146

Quantum dots 97

Quantum noise 48, 81

Quantum size effects 98

Quantum wells 266

Quantum-well heterostructures 97

$\alpha$-quartz 149, 252

Quasi-free electrons 54

Quasi-thermal distribution 299

Quasi-thermal equilibrium 313

Radiation damage 192

Radiationless transition 279

Radio frequency mixer 186,223

Raman echos 83

Raman fiber soliton laser 101

Raman gain 76,98

Raman-induced Kerr effect 68

Raman spectra $364,379,398,404,406$

Raman-type transitions 69,242

Rayleigh-type mixing 87

$\mathrm{Rb}$ vapor 56

Recombination of $\mathrm{I}_{2} 328$

Recombination lifetime 146

Redistribution time 295

Regenerative pulsation 92

Relaxation process 55

Relaxation in solids 254

Relaxation of vibronic levels 314

Renormalization of band gap 131

Resonance enhancement 61

Resonance of nonlinear susceptibility 64

Resonant pulse propagation 262
Rhodamine 6G 55, 72, 95

Rhodopsin 396

Ruby 72

Sampling gate 186

Saturation of absorption 138

Saturation intensity 283

Screening by free carriers and excitons 127

Screening of Fröhlich interaction 137

Second harmonic 96

- generation 36, 98-99

- probing 53

- pulse shape 39

Second-order susceptibility 36

Self-diffraction 86,89

Self-phase modulation $49,82,90,91$

SEPI (short excitation and prolonged interrogation spectroscopy) 260

$\mathrm{SF}_{6} \quad 77$

Signal and idler 47

Silica glass fiber 99

Silicon (Si) 87, 161

Silicon-on-sapphire 173

Silver (Ag) 94, 95

Silver thiagollate $\left(\mathrm{AgGaS}_{2}\right) \quad 46$

Single-photon resonance 68

Single-shot autocorrelation technique 58

Single-shot pulse analysis 73

Single-shot technique 57,60

Singlet oxygen 338

Small-area pulse propagation 83

Small molecular limit 289

Smoluchowski limit 322

Sodium vapor 62

Solitons 99, 100

Solvent effects 319

Solvent polarity 327

Specific heat of molecule 298

State-selective chemistry 279

Stilbene $324,325,327$

Stimulated amplification $47,68,73,78$

Stimulated anti-Stokes Raman scattering 74

Stimulated Brillouin scattering 78

Stimulated electronic Raman scattering 80

Stimulated four-photon generation 70

Stimulated four-wave parametric

amplification 99

Stimulated four-wave parametric emission 69

Stimulated parametric devices 44

Stimulated parametric emission 47

Stimulated parametric generator 96

Stimulated Raman amplifier 77 
Stimulated Raman excitation 280

Stimulated Raman generator 77

Stimulated Raman interaction 101

Stimulated Raman oscillator 77

Stimulated Raman scattering 59, 73, 98, 244

Stokes-anti-Stokes coupling 74

Stokes pulse 77

Streak camera 14, 228

Subharmonic generation 92

Sum-frequency generation $40,42,54,56$, 60

Sum-frequency spectrum 96

Superbroadening 90

Supercontinuum 91

Supercooling 161

Superheating of solid 176

Surface melting 164

Surface polariton 95

Surface second harmonic generation 94 , 168

Switching 185

Synchronous mode-locking 13

Synchronous pumping 8

$T_{1}$ see Energy relaxation time

$T_{2}$ see Phase relaxation time

Tellurium (Te) 54

Temperature tuning 49

Thermal model 160

Thermal population 301

Thermodynamic equilibrium 301

Third harmonic 52,63

- generation 43,60, 94

Third-order autocorrelation 61

Third-order nonlinear susceptibility $\chi^{(3)}$ $36,60,97$

Three-beam grating technique 88

Three-dimensional phase-matching 84,89

Three-pulse echo 267

Time-correlated transmission measurements. 138

Time-domain metrology 213

Time-of-flight method 175

Time-resolved CARS 76

Time-resolved photoluminescence 133

Time-resolved reflectivity measurements 164

Toluene 258

Transient absorption 297

Transient amplification 75

Transient fluorescence excitation 298

Transient frequency-domain experiments 259

Transient grating 265
Transient hot band 298

Transient hot molecule 301

Transient internal temperature 298

Transient refractive index 67

Transient vibrational distribution of energy 297

Traveling-wave excitation 23

Traveling-wave Pockel's cell 227

Trigger jitter 212

Tuning range 44,52

Twisted internal charge transfer 326

Two-beam grating 86

Two-color echo 271

Two-dimensional carrier confinement 144

Two-level molecules 84

Two-level system 85

Two-photon absorption 41, 54

Two-photon fluorescence 57

Two-photon resonance 70

Two-photon transition 305

Two-pulse echo 265

Two-pulse fluorescence 282

Two-pulse infrared spectrum 299

Two-step process 305

Ultrafast chemical reactions 319

Ultrafast phase transformations 159

"Umklapp" third-harmonic generation 61

Up-conversion $44,54,56,59,63,95$

UV pulses 42

Vacuum ultraviolet 62

Velocity overshoot 191

Vibrational dephasing time $T_{2} \quad 279$

Vibrational distribution 297

Vibrational excitation 280

Vibrational heating by electronic excitation 300

Vibrational redistribution $279,294,297$. 299

- in azulene 300

Vibrational relaxation 314

Vibrational-resonant sum-frequency generation 95

Vibrational states 301

Vibrational temperature 296

Visual pigments 401

VT-processes 311

VV-transfer 311

Walk-off angle 38

Water 65, 69

Wave vector mismatch 61

Zero-point fluctuation 68,78

$\mathrm{ZnSe}$ 91, 116, 252 